\title{
Control Bifurcations
}

\author{
Arthur J. Krener, Fellow, IEEE, Wei Kang, Senior Member, IEEE, and Dong Eui Chang
}

\begin{abstract}
A parametrized nonlinear differential equation can have multiple equilibria as the parameter is varied. A local bifurcation of a parametrized differential equation occurs at an equilibrium where there is a change in the topological character of the nearby solution curves. This typically happens because some eigenvalues of the parametrized linear approximating differential equation cross the imaginary axis and there is a change in stability of the equilibrium. The topological nature of the solutions is unchanged by smooth changes of state coordinates so these may be used to bring the differential equation into Poincaré normal form. From this normal form, the type of the bifurcation can be determined. For differential equations depending on a single parameter, the typical ways that the system can bifurcate are fully understood, e.g., the fold (or saddle node), the transcritical and the Hopf bifurcation. A nonlinear control system has multiple equilibria typically parametrized by the set value of the control. A control bifurcation of a nonlinear system typically occurs when its linear approximation loses stabilizability. The ways in which this can happen are understood through the appropriate normal forms. We present the quadratic and cubic normal forms of a scalar input nonlinear control system around an equilibrium point. These are the normal forms under quadratic and cubic change of state coordinates and invertible state feedback. The system need not be linearly controllable. We study some important control bifurcations, the analogues of the classical fold, transcritical and Hopf bifurcations.
\end{abstract}

Index Terms-Control bifurcation, fold control bifurcation, Hopf control bifurcation, normal form, transcritical control bifurcation.

\section{INTRODUCTION}

$\mathbf{T}$ HE theory of normal forms and bifurcations of a parametrized dynamical system is well known [15]. One considers a smooth vector field

$$
\dot{x}=f(x, \mu)
$$

depending on a parameter $\mu$. The equilibria of the vector field are those $x_{e}, \mu_{e}$ such that $f\left(x_{e}, \mu_{e}\right)=0$. Perhaps the most important property of an equilibrium is its stability. In the first approximation, this is determined by the stability of its linear approximating system around $x_{e}, \mu_{e}$

$$
\dot{\delta x}=\frac{\partial f}{\partial x}\left(x_{e}, \mu_{e}\right) \delta x
$$

If all the eigenvalues of $(\partial f / \partial x)\left(x_{e}, \mu_{e}\right)$ lie in the open left half plane then the system (1.1) is locally asymptotically stable

Manuscript received March 29, 2001; revised May 15, 2002 and June 30, 2003. Recommended by Associate Editor Hua Wang.

A. J. Krener is with the Department of Mathematics, University of California, Davis, CA 95616 USA (e-mail: ajkrener@ucdavis.edu).

W. Kang is with the Department of Mathematics, Naval Postgraduate School, Monterey, CA 93940 USA.

D. E. Chang is with the Control and Dynamical Systems, California Institute of Technology, Pasadena, CA 91125 USA

Digital Object Identifier 10.1109/TAC.2004.832199 around the $x_{e}, \mu_{e}$. If one or more eigenvalues lie in the open right-half plane then the system (1.1) is unstable. If all the eigenvalues lie in the closed left-half plane but some are on the imaginary axis then the first approximation is not decisive, (1.1) may be locally asymptotically stable or unstable, depending on higher degree terms.

The topological character of the equilibria can change at a critical value of the parameter, perhaps two branches of equilibria cross or a branch loses or gains stability. Such a state and parameter is called a bifurcation point of the parametrized vector field. A local bifurcation takes place at a parameter value where the system loses structural stability with respect to parameter variations, i.e., the phase portrait around the equilibrium at the critical parameter value is not locally topologically conjugate to the phase portraits around the equilibria at nearby parameter values. If the local linearizations at two equilibria have no poles on the imaginary axis, the same number of strictly stable and the same number of strictly unstable poles then the local phase portraits are topologically conjugate. Therefore a bifurcation is characterized mathematically by one or more eigenvalues of the linearized system crossing the imaginary axis. We restrict our discussion to local bifurcations which we refer to as bifurcations.

A standard approach to analyzing the behavior of the parametrized ordinary differential equations (ODE) (1.1) around a bifurcation point is to treat the parameter as an additional state variable with dynamics $\dot{\mu}=0$ and to compute the center manifold of the extended dynamics through the bifurcation point and the dynamics restricted to this manifold [15]. The center manifold is an invariant manifold of the differential equation which is tangent at the bifurcation point to the eigenspace of the neutrally stable eigenvalues. In practice, one does not compute the center manifold and its dynamics exactly, in most cases of interest, an approximation of degree two or three suffices. If the other eigenvalues are in the open left-half plane, then this part of the dynamics is locally asymptotically stable and therefore can be neglected in a local stability analysis around the bifurcation point. The bifurcation point will be locally asymptotically stable for the complete dynamics iff the dynamics on the center manifold is locally asymptotically stable. Of course, at some nearby equilibria the dynamics may be unstable.

The next step is to compute the Poincare normal form of the center manifold dynamics. From its normal form the bifurcation is recognized and understood. Familiar examples are the fold (or saddle node), the transcritical and the Hopf bifurcations. The first two of these depend on the normal form of degree two and the last one depends on the normal form of degree three. The fold and Hopf bifurcations are the only ones that are generic and of codimension 1, i.e., robust with respect to perturbations and depend on a single parameter, so these are the most important. 
The study of bifurcations of differential equations with control was initiated by Abed and Fu [1], [2]. They considered systems where the parameter is distinct from the control

$$
\dot{x}=f(x, u, \mu) .
$$

They assumed that the uncontrolled system $u=0$ undergoes a bifurcation at a critical value of the parameter $\mu_{0}$ and they studied the stabilizability of the system by quadratic and cubic feedbacks. More general problems of both bifurcation and chaos for control systems are addressed in [3]. In [7], a variety of feedback design methods are introduced for the control of bifurcation and chaos, including the delay or stabilization of bifurcation, and the activation of chaos by feedback control. A survey of theories, methods, and applications of bifurcation control can be found in [8].

Kang [12] studied the degree two normal forms and bifurcations of control systems with (1.3) and without a parameter (1.4). A control system does not need a parameter to bifurcate, the control can play the same role. The equilibria of a controlled differential equation

$$
\dot{x}=f(x, u)
$$

are those values of $x_{e}, u_{e}$ such that $f\left(x_{e}, u_{e}\right)=0$. The equilibria are conveniently parametrized by $u$ or one or more state variables. Two key facts differentiate bifurcations of a control system (1.4) from that of a parametrized system (1.1). The first is that for the latter the structural stability of the equilibria is the crucial issue but for the former the stabilizability by state feedback is the crucial issue. A control system (1.4) is linearly controllable (linearly stabilizable) at $x_{e}, u_{e}$ if the local linear approximation

$$
\dot{\delta x}=\frac{\partial f}{\partial x}\left(x_{e}, u_{e}\right) \delta x+\frac{\partial f}{\partial u}\left(x_{e}, u_{e}\right) \delta u
$$

is controllable (stabilizable). If the linear approximation is stabilizable, then the nonlinear system is locally stabilizable. If the linear approximation is not stabilizable, then the nonlinear system may or may not be locally stabilizable, depending on higher degree terms.

A control system (1.4) is locally, parameterically, smoothly stabilizable at an equilibrium $x_{0}, u_{0}$ if there exists a continuous feedback

$$
u=\kappa\left(x ; x_{e}, u_{e}\right)
$$

which locally, asymptotically stabilizes the system to any nearby equilibrium $x_{e}, u_{e}$. The feedback must be smooth with respect to the current state $x$ and continuous with respect to the target equilibrium $x_{e}, u_{e}$. A control bifurcation of (1.4) takes place at an equilibrium which is not locally, parameterically, smoothly stabilizable. An equilibrium which is linearly stabilizable is also locally, parameterically, smoothly stabilizable but the converse need not hold, e.g.,

$$
\dot{x}=-u^{2} x-x^{3} .
$$

Notice that this is different from the bifurcation of a parametrized system (1.1) which take place at an equilibrium where there is a loss of structural stability with respect to parameter variations. Frequently this loss of structural stability is caused by a loss of linear stability, one or more eigenvalues of (1.2) crossing the imaginary axis. To emphasize this distinction we will refer to the bifurcation of a parametrized system (1.1) as a classical bifurcation.

The other difference between control and classical bifurcations is that when bringing the control system into normal form, a different group of transformations is used. For classical bifurcations, we use parameter dependent change of state coordinates and change of parameter coordinates but for control bifurcations we use change of state coordinates and state dependent change of control coordinates (invertible state feedback) to simplify the dynamics. Such transformations leave unchanged the property of being locally, parameterically, smoothly stabilizable.

After extending Kang's work on the normal forms of degree two, scalar input control systems, we consider degree three normal forms. Similar results for discrete time systems can be found in [5], [16], and [14]. Kang studied the control theoretic analogue of the transcritical bifurcation. We extend this to a study of the control theoretic analogues of the fold and the Hopf bifurcations. We will study the stabilizability of these around the bifurcation point. We will also discuss the parametrized stabilizability of the parametrized family to nearby equilibria by a parametrized control law. A preliminary version of these results was presented in [6] and related work can be found in [19] and [9].

\section{QUADRATIC NORMAL FORMS}

The normal form of control systems is developed in this paper in a different way from that usually found in the literature. Instead of an existence proof, the derivation of normal forms in this paper is constructive. The normal form can be constructed based on two fundamental operations, namely pull up and push down. Furthermore, multiple uncontrollable modes are considered in this paper, which is a larger family of system than those addressed in [12] and [13]. Consider a smooth $\left(C^{\infty}\right)$ control system (1.4) where $x$ is $n$ dimensional, $u$ is one dimensional and $f(0,0)=0$. It is well known that by linear change of state coordinates and linear state feedback, the system can be brought to the form

$$
\begin{aligned}
{\left[\begin{array}{c}
\dot{x}_{1} \\
\dot{x}_{2}
\end{array}\right]=\left[\begin{array}{cc}
A_{1} & 0 \\
0 & A_{2}
\end{array}\right]\left[\begin{array}{l}
x_{1} \\
x_{2}
\end{array}\right]+\left[\begin{array}{c}
0 \\
B_{2}
\end{array}\right] u } \\
+\left[\begin{array}{l}
f_{1}^{[2]}\left(x_{1}, x_{2}, u\right) \\
f_{2}^{[2]}\left(x_{1}, x_{2}, u\right)
\end{array}\right]+O\left(x_{1}, x_{2}, u\right)^{3}
\end{aligned}
$$

where $x_{1}$ and $x_{2}$ are $n_{1}$ and $n_{2}$ dimensional, $n_{1}+n_{2}=n, A_{1}$ is in Jordan form, $A_{2}, B_{2}$ are in controller (Brunovsky) form,, and $f_{i}^{[d]}\left(x_{1}, x_{2}, u\right)$ is a vector field which is a homogeneous polynomial of degree $d$ in its arguments. The linear change of coordinates that brings $A_{1}$ to Jordan form may be complex in which case some of the coordinates $x_{1, i}$ are complex. The complex coordinates come in conjugate pairs. The corresponding $f_{1, i}^{[d]}$ are 
complex valued and come in conjugate pairs. Alternatively, one can use real Jordan forms.

A pair $A_{2}, B_{2}$ is in controller form if

$$
A_{2}=\left[\begin{array}{ccccc}
0 & 1 & 0 & \cdots & 0 \\
0 & 0 & 1 & \cdots & 0 \\
& & \ddots & \ddots & \\
0 & 0 & 0 & \cdots & 1 \\
0 & 0 & 0 & \cdots & 0
\end{array}\right] B_{2}=\left[\begin{array}{c}
0 \\
0 \\
\vdots \\
0 \\
1
\end{array}\right]
$$

We are interested in the normal forms and invariants of the quadratic part of the system under the group of quadratic change of coordinates and an invertible quadratic feedback

$$
\begin{aligned}
{\left[\begin{array}{l}
z_{1} \\
z_{2}
\end{array}\right] } & =\left[\begin{array}{l}
x_{1} \\
x_{2}
\end{array}\right]-\left[\begin{array}{l}
\phi_{1}^{[2]}\left(x_{1}, x_{2}\right) \\
\phi_{2}^{[2]}\left(x_{1}, x_{2}\right)
\end{array}\right] \\
v & =u-\alpha_{1}^{[2]}\left(x_{1}, x_{2}, u\right) .
\end{aligned}
$$

These transformations are only considered where they are invertible, that is, locally around $x=0, u=0$. It is known that the uncontrollable eigenvalues of the linearization of a control system are invariant under linear change of coordinates and linear feedback. Similarly, the quadratic invariants derived in this section are numbers associated with a control system that are invariant under quadratic transformation (2.2)-(2.3). We need notation to describe some of the invariants. Let $X, Y$ be first order partial differential operators in $x_{1}, x_{2}, u$ and $h\left(x_{1}, x_{2}, u\right)$ be a smooth function. The Lie bracket $[X, Y]$ is the partial differential operator defined by

$$
\begin{array}{r}
{[X, Y](h)\left(x_{1}, x_{2}, u\right)=X(Y(h))\left(x_{1}, x_{2}, u\right)} \\
-Y(X(h))\left(x_{1}, x_{2}, u\right) .
\end{array}
$$

It is a simple calculation to show that $[X, Y]$ is also a first order partial differential operator. The iterated Lie bracket is defined for $k \geq 0$ by

$$
\begin{aligned}
\operatorname{ad}_{X}^{0}(Y) & =Y \\
\operatorname{ad}_{X}^{k}(Y) & =\left[X, \mathbf{a d}_{X}^{k-1}(Y)\right] .
\end{aligned}
$$

Define the first-order partial differential operators

$$
\begin{aligned}
F & =\sum_{i=1}^{2} \sum_{j=1}^{n_{i}} f_{i, j}\left(x_{1}, x_{2}, u\right) \frac{\partial}{\partial x_{i, j}} \\
G & =\frac{\partial}{\partial u}
\end{aligned}
$$

where $f_{i, j}$ is the right side of (2.1).

The following theorem extends a result of [12] and [11] to general systems with multiple uncontrollable modes.

Theorem 2.1: Consider (2.1) where $A_{1}$ is in Jordan form, $A_{2}, B_{2}$ are in Brunovsky form and the input is scalar. There exist a quadratic change of coordinates (2.2) and a quadratic feedback (2.3) which transforms the system into the quadratic normal form

$$
\begin{aligned}
& {\left[\begin{array}{c}
\dot{z}_{1} \\
\dot{z}_{2}
\end{array}\right]=\left[\begin{array}{cc}
A_{1} & 0 \\
0 & A_{2}
\end{array}\right]\left[\begin{array}{c}
z_{1} \\
z_{2}
\end{array}\right]+\left[\begin{array}{c}
0 \\
B_{2}
\end{array}\right] v} \\
& +\left[\begin{array}{ccccc}
\tilde{f}_{1}^{[2 ; 0]} & + & \tilde{f}_{1}^{[1 ; 1]} & + & \tilde{f}_{1}^{[0 ; 2]} \\
0 & + & 0 & + & \tilde{f}_{2}^{[0 ; 2]}
\end{array}\right]+O\left(z_{1}, z_{2}, v\right)^{3}
\end{aligned}
$$

where $\tilde{f}_{i}^{\left[d_{1} ; d_{2}\right]}=\tilde{f}_{i}^{\left[d_{1} ; d_{2}\right]}\left(z_{1} ; z_{2}, v\right)$ denotes a polynomial vector field homogeneous of degree $d_{1}$ in $z_{1}$ and homogeneous of degree $d_{2}$ in $z_{2}, v$. The vector field $\tilde{f}_{1}^{[2 ; 0]}$ is in the quadratic normal form of Poincaré [15]

$$
\tilde{f}_{1}^{[2 ; 0]}=\sum_{i=1}^{n_{1}} \sum_{\substack{1 \leq j \leq k \leq n_{1} \\ \lambda_{j}+\lambda_{k}=\lambda_{i}}} \beta_{i}^{j k} \mathbf{e}^{1, i} z_{1, j} z_{1, k}
$$

where $\mathbf{e}^{r, i}$ is the $i$ th unit vector in $z_{r}$ space and $z_{r, i}$ is the $i$ th component of $z_{r}$. The other vector fields are as follows:

$$
\begin{aligned}
\tilde{f}_{1}^{[1 ; 1]} & =\sum_{i=1}^{n_{1}} \sum_{j=1}^{n_{1}} \gamma_{i}^{j 1} \mathbf{e}^{1, i} z_{1, j} z_{2,1} \\
\tilde{f}_{1}^{[0 ; 2]} & =\sum_{i=1}^{n_{1}} \sum_{j=1}^{n_{2}+1} \delta_{i}^{j j} \mathbf{e}^{1, i} z_{2, j}^{2} \\
\tilde{f}_{2}^{[0 ; 2]} & =\sum_{i=1}^{n_{2}-1} \sum_{j=i+2}^{n_{2}+1} \epsilon_{i}^{j j} \mathbf{e}^{2, i} z_{2, j}^{2}
\end{aligned}
$$

where for notational convenience we have defined $z_{2, n_{2}+1}=v$. The vector field, $\tilde{f}_{2}^{[0 ; 2]}$, is in quadratic controller form [11].

If $A_{1}$ is diagonal the normal form is unique, that is, each system in linear normal form (2.1) can be transformed into only one such quadratic normal form (2.6)-(2.10) by quadratic change of coordinates (2.2) and quadratic feedback (2.3). This follows from the fact that when $A_{1}$ is diagonal the numbers in the above, $\beta_{i}^{j k}, \gamma_{i}^{j 1}, \delta_{i}^{j j}, \epsilon_{i}^{j j}$ for the indicated indices, are moduli, i.e., continuous invariants of (2.1) under quadratic change of coordinates and quadratic feedback. Let $\sigma_{j k}=2$ if $j=k$ and $\sigma_{j k}=1$, otherwise. The moduli are as follows:

$$
\begin{aligned}
& \beta_{i}^{j k}=\frac{1}{\sigma_{j k}} \frac{\partial^{2} f_{1, i}}{\partial x_{1, j} \partial x_{1, k}}(0,0,0) \\
& \text { for } 1 \leq i \leq n_{1}, \quad 1 \leq j \leq k \leq n_{1} \\
& \text { and } \lambda_{i}=\lambda_{j}+\lambda_{k} \\
& \gamma_{i}^{j 1}=\sum_{k=1}^{n_{2}+1}\left(\lambda_{i}-\lambda_{j}\right)^{k-1} \frac{\partial^{2} f_{1, i}}{\partial x_{1, j} \partial x_{2, k}}(0,0,0) \\
& \text { for } 1 \leq i, \quad j \leq n_{1} \\
& \delta_{i}^{j j}=\frac{1}{2}\left[\operatorname{ad}_{F}^{k+1}(G), \operatorname{ad}_{F}^{k}(G)\right]\left(x_{1, i}\right)(0,0,0) \text {, } \\
& \text { where } k=n_{2}+1-j \\
& \text { for } 1 \leq i \leq n_{1}, \quad 1 \leq j \leq n_{2}+1 \\
& \epsilon_{i}^{j j}=\frac{1}{2}\left[\operatorname{ad}_{F}^{k+1}(G), \operatorname{ad}_{F}^{k}(G)\right]\left(x_{2, i}\right)(0,0,0) \text {, } \\
& \text { where } k=n_{2}+1-j \\
& \text { for } 1 \leq i \leq n_{2}-1, \quad i+2 \leq j \leq n_{2}+1 \text {. }
\end{aligned}
$$

Remarks: If some of the eigenvalues of $A_{1}$ are complex then a linear complex change of coordinates is required to bring it to Jordan form. In this case some of the coordinates of $z_{1}$ are complex conjugate pairs and some of the coefficients in the normal form are complex. These complex coefficients occur in conjugate pairs so that the real dimension of the coefficient space of the normal form is unchanged.

Regardless of whether the Jordan form of $A_{1}$ is diagonal or not, the numbers $\delta_{i}^{j j}, \epsilon_{i}^{j j}$ are moduli, i.e., invariants under quadratic changes of coordinates and invertible feedbacks. 
Below we shall give an example where $A_{1}$ is not diagonal and the normal form is not unique as not all the $\beta_{i}^{j k}$ are invariants. The linearly controllable part of the system is assumed to be in controller form, which is preferred for feedback design.

The linear change of coordinates and feedback that carries a system into linear normal form (2.1) are not unique. The $x_{1}$ coordinate can be multiplied by an invertible diagonal matrix and the $x_{2}, u$ coordinates can be multiplied by a nonzero scalar without changing the linear normal form (2.1). These extra degrees of freedom do affect the quadratic and higher terms. Tall and Respondek [19] analyzed this for systems where there are no linearly uncontrollable modes.

The assumption of $C^{\infty}$ smoothness is used only in the definitions of (2.13) and (2.14) and the proof that they are moduli. It could be replaced by $C^{r}$ smoothness with $r$ sufficiently large so that the bracketed expressions (2.13), (2.14), which involve partial derivatives, can be calculated. The rest of the theorem requires only $C^{3}$ smoothness.

Proof: We can expand the change of coordinates and feedback as follows:

$$
\begin{aligned}
{\left[\begin{array}{l}
z_{1} \\
z_{2}
\end{array}\right]=} & {\left[\begin{array}{l}
x_{1} \\
x_{2}
\end{array}\right]-\left[\begin{array}{l}
\phi_{1}^{[2 ; 0]}\left(x_{1} ; x_{2}\right) \\
\phi_{2}^{[2 ; 0]}\left(x_{1} ; x_{2}\right)
\end{array}\right] } \\
& -\left[\begin{array}{c}
\phi_{1}^{[1 ; 1]}\left(x_{1} ; x_{2}\right) \\
\phi_{2}^{[1 ; 1]}\left(x_{1} ; x_{2}\right)
\end{array}\right]-\left[\begin{array}{c}
\phi_{1}^{[0 ; 2]}\left(x_{1} ; x_{2}\right) \\
\phi_{2}^{[0 ; 2]}\left(x_{1} ; x_{2}\right)
\end{array}\right] \\
v= & u-\alpha^{[2 ; 0]}\left(x_{1} ; x_{2}, u\right) \\
& -\alpha^{[1 ; 1]}\left(x_{1} ; x_{2}, u\right)-\alpha^{[0 ; 2]}\left(x_{1} ; x_{2}, u\right) .
\end{aligned}
$$

These do not change the linear part of the dynamics. The quadratic part of the dynamics is changed to

$$
\begin{aligned}
\tilde{f}_{i}^{\left[d_{1} ; d_{2}\right]}\left(z_{1} ; z_{2}, v\right) \\
=f_{i}^{\left[d_{1} ; d_{2}\right]}\left(z_{1} ; z_{2}, v\right) \\
\quad-\frac{\partial \phi_{i}^{\left[d_{1} ; d_{2}\right]}}{\partial z_{1}}\left(z_{1} ; z_{2}\right) A_{1} z_{1}-\frac{\partial \phi_{i}^{\left[d_{1} ; d_{2}\right]}}{\partial z_{2}}\left(z_{1} ; z_{2}\right) A_{2} z_{2} \\
\quad+A_{i} \phi_{i}^{\left[d_{1} ; d_{2}\right]}\left(z_{1} ; z_{2}\right)+B_{i} \alpha^{\left[d_{1} ; d_{2}\right]}\left(z_{1} ; z_{2}, v\right)
\end{aligned}
$$

where $B_{1}=0$, so each of the six terms $\tilde{f}_{i}^{\left[d_{1} ; d_{2}\right]}, i=1,2 ; d_{1}=$ $0,1,2 ; d_{2}=2-d_{1}$ can be considered separately

$$
\tilde{f}_{2}^{[0 ; 2]}\left(z_{1} ; z_{2}, v\right)
$$

We start by showing that $\tilde{f}_{2}^{[0 ; 2]}\left(z_{1} ; z_{2}, v\right)$ can be brought into the aforementioned form. There are two basic operations, pull up and push down, which are used to achieve this. Consider a part of the dynamics

$$
\begin{aligned}
\dot{z}_{2, i-1} & =z_{2, i}+\cdots \\
\dot{z}_{2, i} & =z_{2, i+1}+c z_{2, j} z_{2, k}+\cdots
\end{aligned}
$$

where $1<i \leq n_{2}, 1 \leq j \leq k \leq n_{2}+1$, recall $z_{2, n_{2}+1}=v$. The $+\cdots$ indicates other quadratic terms and higher terms. The other quadratic terms will not be changed by the operations that we will do. The higher terms may be changed but we are not interested in them.
If $j<k-1$ we can pull up the quadratic term by defining

$$
\bar{z}_{2, i}=z_{2, i}-c z_{2, j} z_{2, k-1}
$$

then the dynamics becomes

$$
\begin{aligned}
\dot{z}_{2, i-1} & =\bar{z}_{2, i}+c z_{2, j} z_{2, k-1}+\cdots \\
\dot{\bar{z}}_{2, i} & =z_{2, i+1}-c z_{2, j+1} z_{2, k-1}+\cdots
\end{aligned}
$$

and all the other quadratic terms remain the same. Notice that in each of the new quadratic terms the two indices are closer together than the two indices of the original quadratic term.

If $j=k-1$ we can pull up the quadratic term by defining

$$
\bar{z}_{2, i}=z_{2, i}-\frac{c}{2} z_{2, j} z_{2, j}
$$

then the dynamics becomes

$$
\begin{aligned}
\dot{z}_{2, i-1} & =\bar{z}_{2, i}+\frac{c}{2} z_{2, j} z_{2, j}+\cdots \\
\dot{\bar{z}}_{2, i} & =z_{2, i+1}+\cdots
\end{aligned}
$$

and all the other quadratic terms remain the same. Notice that the two indices of the new quadratic term are identical.

Notice also that in either case if $i=1$ then we can still pull up and there is no $z_{2, i-1}$ dynamics to be concerned with so a term disappears.

By pulling up all the quadratic terms until the two indices are equal, we obtain

$$
\dot{z}_{2, i}=z_{2, i+1}+\sum_{j=1}^{n_{2}+1} \epsilon_{i}^{j j} z_{2, j}^{2}+\cdots
$$

which is almost the normal form (2.10). We need to show that $\epsilon_{i}^{j j}$ can be eliminated for $j \leq i+1$.

This is accomplished by the other operation on the dynamics, push down. Consider a piece of the dynamics

$$
\begin{aligned}
\dot{z}_{2, i} & =z_{2, i+1}+c z_{2, j} z_{2, k}+\cdots \\
\dot{z}_{2, i+1} & =z_{2, i+2}+\cdots
\end{aligned}
$$

where $1 \leq i<n_{2}$ and $1 \leq j \leq k \leq n_{2}$. Define

$$
\bar{z}_{2, i+1}=z_{2, i+1}+c z_{2, j} z_{2, k}
$$

yielding

$$
\begin{aligned}
\dot{z}_{2, i}= & \bar{z}_{2, i+1}+\cdots \\
\dot{\bar{z}}_{2, i+1}= & z_{2, i+2}+c z_{2, j+1} z_{2, k} \\
& +c z_{2, j} z_{2, k+1}+\cdots
\end{aligned}
$$

and all the other quadratic terms remain unchanged. Notice that if $i+1=n_{2}$ then we can absorb any quadratic terms into the control using feedback. The terms in (2.16) where $1 \leq j \leq k \leq$ $i+1$ can be pushed down repeatedly and absorbed in the control. The result is (2.10).

We defer to later the proof that the numbers $\epsilon_{i}^{j j}$ in (2.10) are given by (2.14) and are invariants.

$$
\tilde{f}_{2}^{[1 ; 1]}\left(z_{1} ; z_{2}, v\right)
$$


The basic operations, pull up and push down, are slightly different. Consider a part of the dynamics

$$
\begin{aligned}
\dot{z}_{2, i-1} & =z_{2, i}+\cdots \\
\dot{z}_{2, i} & =z_{2, i+1}+c z_{1, j} z_{2, k}+\cdots
\end{aligned}
$$

where $1<i \leq n_{2}, 1 \leq j \leq n_{1}, 1 \leq k \leq n_{2}+1$.

If $1<k$, we can pull up the quadratic term by defining

$$
\bar{z}_{2, i}=z_{2, i}-c z_{1, j} z_{2, k-1}
$$

then the dynamics becomes

$$
\begin{aligned}
\dot{z}_{2, i-1} & =\bar{z}_{2, i}+c z_{1, j} z_{2, k-1}+\cdots \\
\dot{\bar{z}}_{2, i} & =z_{2, i+1}-c\left(\lambda_{j} z_{1, j}+z_{1, j+}\right) z_{2, k-1}+\cdots
\end{aligned}
$$

and all the other quadratic terms remain the same. The symbol $z_{1, j+}$ denotes $z_{1, j+1}$ if

$$
\dot{z}_{1, j}=\lambda_{j} z_{1, j}+z_{1, j+1}
$$

and denotes 0 if

$$
\dot{z}_{1, j}=\lambda_{j} z_{1, j}
$$

Again, if $i=1$, we can still pull up and one of the terms disappears. So by pulling up all quadratic terms until $k=1$, we obtain

$$
\dot{z}_{2, i}=z_{2, i+1}+\bar{c} z_{1, j} z_{2,1}+\cdots
$$

Repeated pushing down eliminates this term. Consider a part of the dynamics

$$
\begin{aligned}
\dot{z}_{2, i} & =z_{2, i+1}+c z_{1, j} z_{2, k}+\cdots \\
\dot{z}_{2, i+1} & =z_{2, i+2}+\cdots
\end{aligned}
$$

where $1 \leq k \leq i \leq n_{2}$. Define

$$
\bar{z}_{2, i+1}=z_{2, i+1}+c z_{1, j} z_{2, k}
$$

yielding

$$
\begin{aligned}
\dot{z}_{2, i}= & \bar{z}_{2, i+1}+\cdots \\
\dot{\bar{z}}_{2, i+1}= & z_{2, i+2}+c\left(\lambda_{j} z_{1, j}+z_{1, j+}\right) z_{2, k} \\
& +c z_{1, j} z_{2, k+1}+\cdots
\end{aligned}
$$

and all the other quadratic terms remain unchanged. Since $k+$ $1 \leq i+1$ we can continue to push down until $i+1=n_{2}$ and the quadratic term can be absorbed into the control using feedback. The result is $\tilde{f}_{2}^{[1 ; 1]}\left(z_{1} ; z_{2}, v\right)=0$

$$
\tilde{f}_{2}^{[2 ; 0]}\left(z_{1} ; z_{2}, v\right)
$$

Consider a part of the dynamics

$$
\begin{aligned}
\dot{z}_{2, i} & =z_{2, i+1}+c z_{1, j} z_{1, k}+\cdots \\
\dot{z}_{2, i+1} & =z_{2, i+2}+\cdots
\end{aligned}
$$

where $1 \leq i \leq n_{2}, 1 \leq j \leq k \leq n_{1}$.
Repeated pushing down eliminates this term. Define

$$
\bar{z}_{2, i+1}=z_{2, i+1}+c z_{1, j} z_{1, k}
$$

yielding

$$
\begin{aligned}
\dot{z}_{2, i}= & \bar{z}_{2, i+1}+\cdots \\
\dot{\bar{z}}_{2, i+1}= & z_{2, i+2}+c\left(\lambda_{j} z_{1, j}+z_{1, j+}\right) z_{1, k} \\
& +c z_{1, j}\left(\lambda_{k} z_{1, k}+z_{1, k+}\right)+\cdots
\end{aligned}
$$

and all the other quadratic terms remain unchanged. The quadratic terms can be pushed down repeatedly until $i+1=n_{2}$ and they can be absorbed in the control via feedback. The result is $\tilde{f}_{2}^{[2 ; 0]}\left(z_{1} ; z_{2}, v\right)=0$

$$
\tilde{f}_{1}^{[2 ; 0]}\left(z_{1} ; z_{2}, v\right)
$$

This is just the quadratic normal form of Poincare as described in the Introduction and $\beta_{i}^{j k}$ are invariants when $A_{1}$ is diagonal. See [4], [10], [15], or [20]. To see this, consider a part of the dynamics

$$
\dot{z}_{1, i}=\lambda_{i} z_{1, i}+c z_{1, j} z_{1, k}+\cdots
$$

where $1 \leq i \leq n_{1}, 1 \leq j \leq k \leq n_{1}$.

If $\lambda_{i} \neq \lambda_{j}+\lambda_{k}$, then define

$$
\bar{z}_{1, i}=z_{1, i}-\frac{c}{\left(\lambda_{j}+\lambda_{k}-\lambda_{i}\right)} z_{1, j} z_{1, k}
$$

so that

$$
\dot{\bar{z}}_{1, i}=\lambda_{i} \bar{z}_{1, i}+\frac{c}{\left(\lambda_{j}+\lambda_{k}-\lambda_{i}\right)}\left(z_{1, j+} z_{1, k}+z_{1, j} z_{1, k+}\right)+\cdots \cdot
$$

We repeat the process until $z_{1, j+}=0$ and $z_{1, k+}=0$.

Next, we show that the numbers $\beta_{i}^{j k}(2.11)$ are invariants when $A_{1}$ is diagonal. Clearly, $\beta_{i}^{j k}$ is possibly changed only by $\phi_{1}^{[2 ; 0]}\left(x_{1} ; x_{2}\right)$. Therefore, we need only consider coordinates changes of the form

$$
\bar{x}_{1, \rho}=x_{1, \rho}+c x_{1, \sigma} x_{1, \tau}
$$

where $1 \leq \rho \leq n_{1}, 1 \leq \sigma \leq \tau \leq n_{1}$ because more general ones are just compositions of these. This coordinate change only affects a piece of the quadratic part of the dynamics (2.1)

$$
\dot{x}_{1, \rho}=\lambda_{\rho} x_{1, \rho}+f_{1, \rho}^{[2]}\left(x_{1}, x_{2}, u\right)+O\left(x_{1}, x_{2}, u\right)^{3}
$$

is transformed to

$$
\begin{aligned}
\dot{\bar{x}}_{1, \rho}=\lambda_{\rho} \bar{x}_{1, \rho}+ & f_{1, \rho}^{[2]}\left(x_{1}, x_{2}, u\right) \\
& +c\left(\lambda_{\sigma}+\lambda_{\tau}-\lambda_{\rho}\right) x_{1, \sigma} x_{1, \tau}+O\left(x_{1}, x_{2}, u\right)^{3} .
\end{aligned}
$$

Clearly, this change only potentially changes $\beta_{i}^{j k}$ when $\rho=$ $i, \sigma=j, \tau=k$, and $\lambda_{i} \neq \lambda_{j}+\lambda_{k}$.

For a system in normal form (2.6), it is straightforward to verify that the $\beta_{i}^{j k}$ of (2.7) is given by (2.11)

$$
\tilde{f}_{1}^{[1 ; 1]}\left(z_{1} ; z_{2}, v\right)
$$


Consider a part of the dynamics

$$
\dot{z}_{1, i}=\lambda_{i} z_{1, i}+z_{1, i+}+c z_{1, j} z_{2, k}+\cdots
$$

where $1 \leq i \leq n_{1}, 1 \leq j \leq n_{1}, 1 \leq k \leq n_{2}+1$.

If $k>1$, then we can pull up by defining

$$
\bar{z}_{1, i}=z_{1, i}-c z_{1, j} z_{2, k-1}
$$

so that

$\dot{\bar{z}}_{1, i}=\lambda_{i} \bar{z}_{1, i}+z_{1, i+}+c\left(\left(\lambda_{i}-\lambda_{j}\right) z_{1, j}-z_{1, j+}\right) z_{2, k-1}+\cdots$

We can continue to pull up until $k=1$. The result is (2.8).

Next, we show that the numbers $\gamma_{i}^{j 1}(2.12)$ are invariants when $A_{1}$ is diagonal. Clearly, they are potentially changed only by $\phi_{1}^{[1 ; 1]}\left(x_{1} ; x_{2}\right)$. Therefore we need only consider coordinates changes of the form

$$
\bar{x}_{1, \rho}=x_{1, \rho}+c x_{1, \sigma} x_{2, \tau}
$$

where $1 \leq \rho, \sigma \leq n_{1}, 1 \leq \tau \leq n_{2}$ because more general ones are just compositions of these. This coordinate change only affects a piece of the dynamics (2.1),

$$
\dot{x}_{1, \rho}=\lambda_{\rho} x_{1, \rho}+f_{1, \rho}^{[2]}\left(x_{1}, x_{2}, u\right)+O\left(x_{1}, x_{2}, u\right)^{3}
$$

is transformed to

$$
\begin{aligned}
\dot{\bar{x}}_{1, \rho}= & \lambda_{\rho} \bar{x}_{1, \rho}+f_{1, \rho}^{[2]}\left(x_{1}, x_{2}, u\right) \\
& +c\left(\lambda_{\sigma}-\lambda_{\rho}\right) x_{1, \sigma} x_{2, \tau}+c x_{1, \sigma} x_{2, \tau+1}+O\left(x_{1}, x_{2}, u\right)^{3} .
\end{aligned}
$$

This potentially affects $\gamma_{i}^{j 1}$ only if $\rho=i$ and $\sigma=j$. The sum (2.12) is unchanged regardless of $\tau$.

For a system in normal form (2.6), it is straightforward to verify that the $\gamma_{i}^{j 1}$ of (2.8) is given by (2.12)

$$
\tilde{f}_{1}^{[0 ; 2]}\left(z_{1} ; z_{2}, v\right) \text {. }
$$

Consider a part of the dynamics

$$
\dot{z}_{1, i}=\lambda_{i} z_{1, i}+z_{1, i+}+c z_{2, j} z_{2, k}+\cdots
$$

where $1 \leq i \leq n_{1}, 1 \leq j \leq k \leq n_{2}$.

If $j<k-1$, then we can pull up by defining

$$
\bar{z}_{1, i}=z_{1, i}-c z_{2, j} z_{2, k-1}
$$

then

$\dot{\bar{z}}_{1, i}=\lambda_{i} \bar{z}_{1, i}+z_{1, i+}+c \lambda_{i} z_{2, j} z_{2, k-1}-c z_{2, j+1} z_{2, k-1}+\cdots$. If $j=k-1$, then we can pull up by defining

$$
\bar{z}_{1, i}=z_{1, i}-\frac{c}{2} z_{2, j} z_{2, j}
$$

then

$$
\dot{\bar{z}}_{1, i}=\lambda_{i} \bar{z}_{1, i}+\frac{c \lambda_{i}}{2} z_{2, j} z_{2, j}+\cdots
$$

We can continue to pull up until the two indices are the same. The result is (2.9).

We show that the numbers $\delta_{i}^{j j}, \epsilon_{i}^{j j}$ as given by (2.13), (2.14) are invariants under quadratic change of coordinates (2.2) and quadratic feedback (2.3) regardless of the Jordan form of $A_{1}$.
Under the quadratic feedback the control is transformed from $u$ to $v$. Let $G$ be defined by (2.5) and

$$
\bar{G}=\frac{\partial}{\partial v} \text {. }
$$

By the chain rule

$$
G=\frac{\partial v}{\partial u} \bar{G}=\left(1+O\left(x_{1}, x_{2}, u\right)\right) \bar{G} .
$$

It is straightforward to show by induction that for $1 \leq k \leq n_{2}$

$$
(-1)^{k} \operatorname{ad}_{F}^{k}(G)=\frac{\partial}{\partial x_{2, n_{2}+1-k}}+O\left(x_{1}, x_{2}, u\right) \frac{\partial}{\partial x}
$$

$$
(-1)^{n_{2}+1} \mathbf{a d}_{F}^{n_{2}+1}(G)=O\left(x_{1}, x_{2}, u\right) \frac{\partial}{\partial x}
$$

and

$$
\begin{aligned}
\operatorname{ad}_{F}^{k}(G)= & \mathbf{a d}_{F}^{k}(\bar{G})+\sum_{r=0}^{k} O\left(x_{1}, x_{2}, u\right) \\
& \times \frac{\partial}{\partial x_{2, n_{2}+1-r}}+O\left(x_{1}, x_{2}, u\right)^{2} \frac{\partial}{\partial x} \\
\operatorname{ad}_{F}^{n_{2}+1}(G)= & \mathbf{a d}_{F}^{n_{2}+1}(\bar{G})+\sum_{r=0}^{n_{2}} O\left(x_{1}, x_{2}, u\right) \\
& \times \frac{\partial}{\partial x_{2, n_{2}+1-r}}+O\left(x_{1}, x_{2}, u\right)^{2} \frac{\partial}{\partial x} .
\end{aligned}
$$

Therefore, for $1 \leq k \leq n_{2}$ and $1 \leq i \leq n_{1}$

$$
\begin{aligned}
{\left[\operatorname{ad}_{F}^{k+1}(G), \operatorname{ad}_{F}^{k}(G)\right] } & \left(x_{1, i}\right)(0,0,0) \\
= & \left.\operatorname{ad}_{F}^{k+1}(\bar{G}), \mathbf{a d}_{F}^{k}(\bar{G})\right]\left(x_{1, i}\right)(0,0,0) .
\end{aligned}
$$

For $1 \leq k \leq n_{2}$ and $1 \leq i \leq n_{2}-1-k$

$$
\begin{aligned}
{\left[\operatorname{ad}_{F}^{k+1}(G), \operatorname{ad}_{F}^{k}(G)\right] } & \left(x_{2, i}\right)(0,0,0) \\
= & {\left[\operatorname{ad}_{F}^{k+1}(\bar{G}), \mathbf{a d}_{F}^{k}(\bar{G})\right]\left(x_{2, i}\right)(0,0,0) . }
\end{aligned}
$$

We have shown that (2.13) and (2.14) are invariant under quadratic feedback (2.3). Since $z=x+O(x)^{2}$ they are also invariant under quadratic change of coordinates (2.2). In other words, for the indicated ranges of indexes

$$
\begin{aligned}
& \frac{1}{2}\left[\operatorname{ad}_{F}^{k+1}(G), \mathbf{a d}_{F}^{k}(G)\right]\left(x_{1, i}\right)(0,0,0) \\
& \quad=\frac{1}{2}\left[\operatorname{ad}_{F}^{k+1}(G), \mathbf{a d}_{F}^{k}(G)\right]\left(z_{1, i}\right)(0,0,0)
\end{aligned}
$$

and

$$
\begin{aligned}
& \frac{1}{2}\left[\operatorname{ad}_{F}^{k+1}(G), \mathbf{a d}_{F}^{k}(G)\right]\left(x_{2, i}\right)(0,0,0) \\
& \quad=\frac{1}{2}\left[\operatorname{ad}_{F}^{k+1}(G), \mathbf{a d}_{F}^{k}(G)\right]\left(z_{2, i}\right)(0,0,0) .
\end{aligned}
$$

It remains to show that if a system is in quadratic normal form (2.6) then $\delta_{i}^{j j}, \epsilon_{i}^{j j}$ are given by (2.13), (2.14). Let

$$
\begin{aligned}
\bar{F} & =\sum_{i=1}^{2} \sum_{j=1}^{n_{i}} \tilde{f}_{i, j}\left(z_{1}, z_{2}, v\right) \frac{\partial}{\partial z_{i, j}} \\
\bar{G} & =\frac{\partial}{\partial v}
\end{aligned}
$$

where $\tilde{f}$ denotes the right-hand side of (2.6). 
By induction, one can show that for $1 \leq k \leq n_{2}, l=n_{2}+$ $1-k$

$$
\begin{aligned}
(-1)^{k} & \mathbf{a d}_{\bar{F}}^{k}(\bar{G}) \\
= & \frac{\partial}{\partial z_{2, l}}+2 \sum_{i=1}^{n_{1}} \delta_{i}^{l+1, l+1} z_{2, l+1} \frac{\partial}{\partial z_{1, i}} \\
& +2 \sum_{i=1}^{l-1} \epsilon_{i}^{l+1, l+1} z_{2, l+1} \frac{\partial}{\partial z_{2, i}} \\
& +O\left(z_{1}, z_{2, l+2}, \ldots, z_{2, n_{2}+1}\right) \frac{\partial}{\partial z}+O(z)^{2} \frac{\partial}{\partial z} \\
(-1)^{n_{2}+1} \mathbf{a d}_{\bar{F}}^{n_{2}+1}(\bar{G}) & \\
= & 2 \sum_{i=1}^{n_{1}} \delta_{i}^{11} z_{2,1} \frac{\partial}{\partial z_{1, i}} \\
& +O\left(z_{1}, z_{2,2}, \ldots, z_{2, n_{2}+1}\right) \frac{\partial}{\partial z}+O(z)^{2} \frac{\partial}{\partial z} .
\end{aligned}
$$

Then, for $l=n_{2}+1-k$

$$
\begin{aligned}
{\left[\operatorname{ad}_{\bar{F}}^{k+1}(\bar{G}), \operatorname{ad}_{\bar{F}}^{k}(\bar{G})\right]=2 \sum_{i=1}^{n_{1}} \delta_{i}^{l, l} } & \frac{\partial}{\partial z_{1, i}} \\
& +2 \sum_{i=1}^{l-2} \epsilon_{i}^{l, l} \frac{\partial}{\partial z_{2, i}}+O(z) \frac{\partial}{\partial z}
\end{aligned}
$$

which confirms (2.13) and (2.14).

Remarks: It is not essential that $A_{2}, B_{2}$ be in Brunovsky form, all they need to be is in controller companion form, i.e.,

$$
\begin{aligned}
A_{2} & =\left[\begin{array}{ccccc}
0 & 1 & 0 & \cdots & 0 \\
0 & 0 & 1 & \cdots & 0 \\
& & \ddots & \ddots & \\
0 & 0 & 0 & \cdots & 1 \\
a_{n, 1} & a_{n, 2} & a_{n, 3} & \cdots & a_{n, n}
\end{array}\right] \\
B_{2} & =\left[\begin{array}{c}
0 \\
0 \\
\vdots \\
0 \\
b_{n}
\end{array}\right] .
\end{aligned}
$$

The quadratic normal form (2.6)-(2.10) remains the same but the moduli (2.11)-(2.14) are different.

According to (2.11), $\beta_{i}^{j k}$ is simply the coefficient of the term $z_{1, j} z_{1, k} \mathbf{e}^{1, i}$. It was proved by Poincaré that the coefficient of $z_{1, j} z_{1, k} \mathbf{e}^{1, i}$ is invariant under quadratic change of coordinates if $\lambda_{i}+\lambda_{j}=\lambda_{i}$. It is called a resonant term.

In the aforementioned normal form, the quadratic terms which are not removable have been pulled up as far as possible, i.e., either to $z_{1, j} z_{2,1}$ or $z_{2,1}^{2}$. One can also push down the unremovable terms in $\tilde{f}_{2}$ to obtain the following normal form [11]:

$$
\begin{aligned}
& {\left[\begin{array}{c}
\dot{z}_{1} \\
\dot{z}_{2}
\end{array}\right]=\left[\begin{array}{cc}
A_{1} & 0 \\
0 & A_{2}
\end{array}\right]\left[\begin{array}{l}
z_{1} \\
z_{2}
\end{array}\right]+\left[\begin{array}{c}
0 \\
B_{2}
\end{array}\right] v} \\
& +\left[\begin{array}{ccccc}
\bar{f}_{1}^{[2 ; 0]} & + & \bar{f}_{1}^{[1 ; 1]} & + & \bar{f}_{1}^{[0 ; 2]} \\
0 & + & 0 & + & \bar{f}_{2}^{0 ; 2]}
\end{array}\right]\left(z_{1}, z_{2}, v\right) \\
& +O\left(z_{1}, z_{2}, v\right)^{3}
\end{aligned}
$$

where

$$
\begin{aligned}
\bar{f}_{1}^{[2 ; 0]} & =\tilde{f}_{1}^{[2 ; 0]} \\
\bar{f}_{1}^{[1 ; 1]} & =\tilde{f}_{1}^{[1 ; 1]} \\
\bar{f}_{1}^{[0 ; 2]} & =\tilde{f}_{1}^{[0 ; 2]} \\
\bar{f}_{2}^{[0 ; 2]} & =\sum_{i=1}^{n_{2}-1} \sum_{j=n_{2}-i+2}^{n_{2}+1} \epsilon_{i}^{j, n_{2}+1} \mathrm{e}^{2, i} z_{2, j} v .
\end{aligned}
$$

It may not be possible to push down the quadratic terms in $\tilde{f}_{1}$ because of resonances.

The normal form is not unique when $A_{1}$ is not diagonal, for example, suppose $n_{1}=2, n_{2}=0$ and

$$
A_{1}=\left[\begin{array}{ll}
0 & 1 \\
0 & 0
\end{array}\right] \text {. }
$$

Since $\lambda_{1}=\lambda_{2}=0$ every quadratic term is resonant but obviously any quadratic term in $\dot{x}_{11}$ can be pushed down into $\dot{x}_{12}$.

\section{CUBic NORMAL Forms}

The following theorem is new.

Theorem 3.1: Consider a smooth $\left(C^{\infty}\right)$ control system (2.1) where $A_{1}$ is in Jordan form, $A_{2}, B_{2}$ are in Brunovsky form and the input is scalar. There exist a quadratic and cubic change of coordinates and a quadratic and cubic feedback

$$
\begin{aligned}
{\left[\begin{array}{l}
z_{1} \\
z_{2}
\end{array}\right] } & =\left[\begin{array}{l}
x_{1} \\
x_{2}
\end{array}\right]-\left[\begin{array}{l}
\phi_{1}^{[2]}\left(x_{1}, x_{2}\right) \\
\phi_{2}^{[2]}\left(x_{1}, x_{2}\right)
\end{array}\right]-\left[\begin{array}{l}
\phi_{1}^{[3]}\left(x_{1}, x_{2}\right) \\
\phi_{2}^{[3]}\left(x_{1}, x_{2}\right)
\end{array}\right] \\
v & =u-\alpha_{1}^{[2]}\left(x_{1}, x_{2}, u\right)-\alpha_{1}^{[3]}\left(x_{1}, x_{2}, u\right)
\end{aligned}
$$

which transforms (2.1) into the cubic normal form

$$
\begin{aligned}
& {\left[\begin{array}{c}
\dot{z}_{1} \\
\dot{z}_{2}
\end{array}\right]=\left[\begin{array}{cc}
A_{1} & 0 \\
0 & A_{2}
\end{array}\right]\left[\begin{array}{l}
z_{1} \\
z_{2}
\end{array}\right]+\left[\begin{array}{c}
0 \\
B_{2}
\end{array}\right] v} \\
& +\left[\begin{array}{ccccc}
\tilde{f}_{1}^{[2 ; 0]} & + & \tilde{f}_{1}^{[1 ; 1]} & + & \tilde{f}_{1}^{[0 ; 2]} \\
0 & + & 0 & + & \tilde{f}_{2}^{[0 ; 2]}
\end{array}\right] \\
& +\left[\begin{array}{ccccccc}
\tilde{f}_{1}^{[3 ; 0]} & + & \tilde{f}_{1}^{[2 ; 1]} & + & \tilde{f}_{1}^{[1 ; 2]} & + & \tilde{f}_{1}^{[0 ; 3]} \\
0 & + & 0 & + & \tilde{f}_{2}^{[1 ; 2]} & + & \tilde{f}_{2}^{[0 ; 3]}
\end{array}\right] \\
& +O\left(z_{1}, z_{2}, v\right)^{4}
\end{aligned}
$$

where the quadratic part is as in the previous theorem. Furthermore, $\tilde{f}_{1}^{[3 ; 0]}$ is in the cubic normal form of Poincaré [15]

$$
\tilde{f}_{1}^{[3 ; 0]}=\sum_{i=1}^{n_{1}} \sum_{\substack{1 \leq j \leq k \leq l \leq n_{1} \\ \lambda_{j}+\lambda_{k}+\lambda_{l}=\lambda_{i}}} \beta_{i}^{j k l} \mathbf{e}^{1, i} z_{1, j} z_{1, k} z_{1, l} .
$$

The other cubic vector fields are as follows:

$$
\begin{aligned}
& \tilde{f}_{1}^{[2 ; 1]}=\sum_{i=1}^{n_{1}} \sum_{j=1}^{n_{1}} \sum_{k=j}^{n_{1}} \gamma_{i}^{j k 1} \mathbf{e}^{1, i} z_{1, j} z_{1, k} z_{2,1} \\
& \tilde{f}_{1}^{[1 ; 2]}=\sum_{i=1}^{n_{1}} \sum_{j=1}^{n_{1}} \sum_{k=1}^{n_{2}+1} \delta_{i}^{j k k} \mathbf{e}^{1, i} z_{1, j} z_{2, k}^{2} \\
& \tilde{f}_{1}^{[0 ; 3]}=\sum_{i=1}^{n_{1}} \sum_{j=1}^{n_{2}+1} \sum_{k=j}^{n_{2}+1} \epsilon_{i}^{j k k} \mathbf{e}^{1, i} z_{2, j} z_{2, k}^{2}
\end{aligned}
$$




$$
\begin{aligned}
\tilde{f}_{2}^{[1 ; 2]} & =\sum_{i=1}^{n_{2}-1} \sum_{j=1}^{n_{1}} \sum_{k=i+2}^{n_{2}+1} \zeta_{i}^{j k k} \mathrm{e}^{2, i} z_{1, j} z_{2, k}^{2} \\
\tilde{f}_{2}^{[0 ; 3]} & =\sum_{i=1}^{n_{2}-1} \sum_{k=i+2}^{n_{2}+1} \sum_{j=1}^{k} \eta_{i}^{j k k} \mathrm{e}^{2, i} z_{2, j} z_{2, k}^{2}
\end{aligned}
$$

If $A_{1}$ is diagonal, then the normal form is unique, that is, each system in quadratic normal form (2.6) can be transformed into only one such cubic normal form (3.3)-(3.9) by cubic change of coordinates (3.1) and cubic feedback (3.2). This follows from the fact that the numbers in the above, $\beta_{i}^{j k l}, \gamma_{i}^{j k 1}, \delta_{i}^{j k k}, \epsilon_{i}^{j k k}$, $\zeta_{i}^{j k k}, \eta_{i}^{j k k}$ for the indicated indexes, are moduli, i.e., continuous invariants of the system (2.6) under cubic change of coordinates and cubic feedback. Let $\sigma_{j k l}=6$ if $j=k=l$ and $\sigma_{j k l}=$ $\sigma_{j k} \sigma_{j l} \sigma_{k l}$, otherwise. The moduli are as follows:

$$
\begin{aligned}
& \beta_{i}^{j k l}=\frac{1}{\sigma_{j k l}} \frac{\partial^{3} f_{1, i}}{\partial x_{1, j} \partial x_{1, k} \partial x_{1, l}}(0,0,0) \\
& \text { for } 1 \leq i, j, \quad k \leq n_{1} \quad \text { and } \\
& \lambda_{i}=\lambda_{j}+\lambda_{k}+\lambda_{l} \\
& \gamma_{i}^{j k 1}=\frac{1}{\sigma_{j k}} \sum_{\emptyset \oplus)}^{n_{2}}\left(\lambda_{i}-\lambda_{j}-\lambda_{k}\right)^{l} \\
& \times \frac{\partial^{3} f_{1, i}}{\partial x_{1, j} \partial x_{1, k} \partial x_{2, l+1}}(0,0,0) \\
& \text { for } 1 \leq i \leq n_{1}, \quad 1 \leq j \leq k \leq n_{1} \\
& \delta_{i}^{j k k}=\frac{1}{2}\left[\frac{\partial}{\partial x_{1, j}},\left[\operatorname{ad}_{F}^{n_{2}+2-k}(G), \operatorname{ad}_{F}^{n_{2}+1-k}(G)\right]\right]\left(x_{1, i}\right)(0,0,0) \\
& \text { for } 1 \leq i, \quad j \leq n_{1} \quad \text { and } \\
& 1 \leq k \leq n_{2}+1 \\
& \epsilon_{i}^{j k k}=\frac{(-1)^{n_{2}+1-j}}{\sigma_{j k k}}\left[\operatorname{ad}_{F}^{n_{2}+1-j}(G),\right. \\
& \left.\left[\operatorname{ad}_{F}^{n_{2}+2-k}(G), \operatorname{ad}_{F}^{n_{2}+1-k}(G)\right]\right]\left(x_{1, i}\right)(0,0,0) \\
& \text { for } 1 \leq i \leq n_{1} \text { and } \\
& 1 \leq j \leq k \leq n_{2}+1 \\
& \zeta_{i}^{j k k}=\frac{1}{2}\left[\frac{\partial}{\partial x_{1, j}},\left[\operatorname{ad}_{F}^{n_{2}+2-k}(G), \operatorname{ad}_{F}^{n_{2}+1-k}(G)\right]\right]\left(x_{2, i}\right)(0,0,0) \\
& \text { for } 1 \leq i \leq n_{2}-1, \quad 1 \leq j \leq n_{1} \quad \text { and } \\
& i+2 \leq k \leq n_{2}+1 \\
& \eta_{i}^{j k k}=\frac{(-1)^{n_{2}+1-j}}{\sigma_{j k k}}\left[\operatorname{ad}_{F}^{n_{2}+1-j}(G),\right. \\
& \left.\left[\operatorname{ad}_{F}^{n_{2}+2-k}(G), \operatorname{ad}_{F}^{n_{2}+1-k}(G)\right]\right]\left(x_{2, i}\right)(0,0,0) \\
& \text { for } 1 \leq i \leq n_{2}-1 \\
& i+2 \leq k \leq n_{2}+1 \quad \text { and } \quad 1 \leq j \leq k
\end{aligned}
$$

where $F, G$ are the partial differential operators (2.4), (2.5).

Remarks: If some of the eigenvalues of $A_{1}$ are complex, then a linear complex change of coordinates is required to bring it to Jordan form. In this case, some of the coordinates of $z_{1}$ are complex conjugate pairs and some of the coefficients in the normal form are complex. These complex coefficients occur in conjugate pairs so that the real dimension of the coefficient space of the normal form is unchanged.
Regardless of whether the Jordan form of $A_{1}$ is diagonal or not, the numbers $\delta_{i}^{j k k}, \epsilon_{i}^{j k k}, \zeta_{i}^{j k k}, \eta_{i}^{j k k}$ are moduli, i.e., invariants under cubic change of coordinates and feedback.

The linear and quadratic change of coordinates and feedback that carries the system into quadratic normal form (2.6) are not unique. These extra degrees of freedom do affect the cubic and higher terms. Tall and Respondek [19] have analyzed this for systems where there are no uncontrollable modes.

The assumption of $C^{\infty}$ smoothness is used only in the definitions of (3.12)-(3.15) and the proof that they are moduli. It could be replaced by $C^{r}$ smoothness with $r$ sufficiently large that the bracketed expressions (3.12)-(3.15) can be calculated. The rest of the theorem requires only $C^{4}$ smoothness.

Proof: We can expand the change of coordinates and feedback as follows:

$$
\begin{aligned}
{\left[\begin{array}{l}
z_{1} \\
z_{2}
\end{array}\right]=} & {\left[\begin{array}{l}
x_{1} \\
x_{2}
\end{array}\right]-\left[\begin{array}{c}
\phi_{1}^{[3 ; 0]}\left(x_{1} ; x_{2}\right) \\
\phi_{2}^{[3 ; 0]}\left(x_{1} ; x_{2}\right)
\end{array}\right]-\left[\begin{array}{c}
\phi_{1}^{[2 ; 1]}\left(x_{1} ; x_{2}\right) \\
\phi_{2}^{[2 ; 1]}\left(x_{1} ; x_{2}\right)
\end{array}\right] } \\
& -\left[\begin{array}{c}
\phi_{1}^{[1 ; 2]}\left(x_{1} ; x_{2}\right) \\
\phi_{2}^{[1 ; 2]}\left(x_{1} ; x_{2}\right)
\end{array}\right]-\left[\begin{array}{c}
\phi_{1}^{[0 ; 3]}\left(x_{1} ; x_{2}\right) \\
\phi_{2}^{[0 ; 3]}\left(x_{1} ; x_{2}\right)
\end{array}\right] \\
v= & u-\alpha^{[3 ; 0]}\left(x_{1} ; x_{2}, u\right)-\alpha^{[2 ; 1]}\left(x_{1} ; x_{2}, u\right) \\
& -\alpha^{[1 ; 2]}\left(x_{1} ; x_{2}, u\right)-\alpha^{[0 ; 3]}\left(x_{1} ; x_{2}, u\right) .
\end{aligned}
$$

These do not change the linear and quadratic parts of the dynamics. The cubic part of the dynamics is changed to

$$
\begin{aligned}
\tilde{f}_{i}^{\left[d_{1} ; d_{2}\right]}\left(z_{1} ; z_{2}, v\right) \\
=f_{i}^{\left[d_{1} ; d_{2}\right]}\left(z_{1} ; z_{2}, v\right) \\
\quad-\frac{\partial \phi_{i}^{\left[d_{1} ; d_{2}\right]}}{\partial z_{1}}\left(z_{1} ; z_{2}\right) A_{1} z_{1}-\frac{\partial \phi_{i}^{\left[d_{1} ; d_{2}\right]}}{\partial z_{2}}\left(z_{1} ; z_{2}\right) A_{2} z_{2} \\
\quad+A_{i} \phi_{i}^{\left[d_{1} ; d_{2}\right]}\left(z_{1} ; z_{2}\right)+B_{i} \alpha^{\left[d_{1} ; d_{2}\right]}\left(z_{1} ; z_{2}, v\right)
\end{aligned}
$$

where $B_{1}=0$, so each of the eight terms $\tilde{f}_{i}^{\left[d_{1} ; d_{2}\right]}, i=1,2$; $d_{1}=0,1,2,3 ; d_{2}=3-d_{1}$ can be considered separately

$$
\tilde{f}_{2}^{[0 ; 3]}\left(z_{1} ; z_{2}, v\right) \text {. }
$$

Consider a part of the dynamics

$$
\begin{aligned}
\dot{z}_{2, i-1} & =z_{2, i}+\tilde{f}_{2, i-1}^{[2]}\left(z_{1}, z_{2}, v\right)+\cdots \\
\dot{z}_{2, i} & =z_{2, i+1}+\tilde{f}_{2, i}^{[2]}\left(z_{1}, z_{2}, v\right)+c z_{2, j} z_{2, k} z_{2, l}+\cdots
\end{aligned}
$$

where $1<i \leq n_{2}, 1 \leq j \leq k \leq l \leq n_{2}+1$, recall $z_{2, n_{2}+1}=v$. The $+\cdots$ stands for other cubic terms and higher degree terms. The other cubic terms will not be affected by the operations that we do and we ignore the higher degree terms.

If $k<l-1$, we can pull up the cubic term by defining

$$
\bar{z}_{2, i}=z_{2, i}-c z_{2, j} z_{2, k} z_{2, l-1}
$$

then the dynamics becomes

$$
\begin{aligned}
\dot{z}_{2, i-1}= & \bar{z}_{2, i}+\tilde{f}_{2, i-1}^{[2]}\left(z_{1}, z_{2}, v\right)+c z_{2, j} z_{2, k} z_{2, l-1}+\cdots \\
\dot{\bar{z}}_{2, i}= & z_{2, i+1}+\tilde{f}_{2, i}^{[2]}\left(z_{1}, z_{2}, v\right)-c z_{2, j+1} z_{2, k} z_{2, l-1} \\
& -c z_{2, j} z_{2, k+1} z_{2, l-1}+\cdots
\end{aligned}
$$

and all the other cubic terms remain the same. Notice that the two largest indices of the new cubic terms are closer together than those of the original cubic term. 
Similarly, if $j<k=l-1$ we can pull up the cubic term by defining

$$
\bar{z}_{2, i}=z_{2, i}-\frac{c}{2} z_{2, j} z_{2, k} z_{2, k}
$$

In the new terms generated by (3.16), the two largest indices of the new cubic terms are identical.

Different from the quadratic normal form, we have to consider the case in which $j=k=l-1$. We can pull up the cubic term by defining

$$
\bar{z}_{2, i}=z_{2, i}-\frac{c}{3} z_{2, j} z_{2, j} z_{2, j}
$$

then the dynamics becomes

$$
\begin{aligned}
\dot{z}_{2, i-1} & =\bar{z}_{2, i}+\tilde{f}_{2, i-1}^{[2]}\left(z_{1}, z_{2}, v\right)+\frac{c}{3} z_{2, j} z_{2, j} z_{2, j}+\cdots \\
\dot{\bar{z}}_{2, i} & =z_{2, i+1}+\tilde{f}_{2, i}^{[2]}\left(z_{1}, z_{2}, v\right)+\cdots
\end{aligned}
$$

and all the other cubic terms remain the same. Notice that all the indexes of the new cubic term are identical.

In any case, if $i=1$ then we can still pull up and there is no $z_{2, i-1}$ dynamics to be concerned with so a term disappears.

By pulling up all the cubic terms until their two largest indexes are identical, we obtain

$$
\dot{z}_{2, i}=z_{2, i+1}+\tilde{f}_{2, i}^{[2]}\left(z_{1}, z_{2}, v\right)+\sum_{k=1}^{n_{2}+1} \sum_{j=1}^{k} \eta_{i}^{j k k} z_{2, j} z_{2, k}^{2}+\cdots
$$

which is almost the normal form (3.9).

By pushing down, we can make $\eta_{i}^{j k k}=0$ for $1 \leq j \leq k \leq$ $i+1$. Consider a piece of the dynamics

$$
\begin{aligned}
\dot{z}_{2, i} & =z_{2, i+1}+\tilde{f}_{2, i}^{[2]}\left(z_{1}, z_{2}, v\right)+c z_{2, j} z_{2, k} z_{2, l}+\cdots \\
\dot{z}_{2, i+1} & =z_{2, i+2}+\tilde{f}_{2, i+1}^{[2]}\left(z_{1}, z_{2}, v\right)+\cdots
\end{aligned}
$$

If $1 \leq j \leq k \leq l \leq n_{2}$, define

$$
\bar{z}_{2, i+1}=z_{2, i+1}+c z_{2, j} z_{2, k} z_{2, l}
$$

yielding

$$
\begin{aligned}
\dot{z}_{2, i}= & \bar{z}_{2, i+1}+\cdots \\
\dot{\bar{z}}_{2, i+1}= & z_{2, i+2}+\tilde{f}_{2, i+1}^{[2]}\left(z_{1}, z_{2}, v\right) \\
& +c z_{2, j+1} z_{2, k} z_{2, l}+c z_{2, j} z_{2, k+1} z_{2, l} \\
& +c z_{2, j} z_{2, k+1} z_{2, l+1}+\cdots
\end{aligned}
$$

and all the other cubic terms remain unchanged. Notice that if $i+1=n_{2}$ then we can absorb the cubic terms into the control using feedback. The terms in (3.16) where $1 \leq j \leq k \leq l \leq$ $i+1$ can be repeatedly pushed down and absorbed in the control. The result is (3.9).

We defer to later the proof that $\eta_{i}^{j k k}$ is given by (3.15) and is an invariant

$$
\tilde{f}_{2}^{[1 ; 2]}\left(z_{1} ; z_{2}, v\right)
$$

Consider a part of the dynamics

$$
\begin{aligned}
\dot{z}_{2, i-1} & =z_{2, i}+\tilde{f}_{2, i-1}^{[2]}\left(z_{1}, z_{2}, v\right)+\cdots \\
\dot{z}_{2, i} & =z_{2, i+1}+\tilde{f}_{2, i}^{[2]}\left(z_{1}, z_{2}, v\right)+c z_{1, j} z_{2, k} z_{2, l}+\cdots
\end{aligned}
$$

where $1<i \leq n_{2}, 1 \leq j \leq n_{1}, 1 \leq k \leq l \leq n_{2}+1$.

If $k<l-1$, we can pull up the cubic term by defining

$$
\bar{z}_{2, i}=z_{2, i}-c z_{1, j} z_{2, k} z_{2, l-1}
$$

then the dynamics becomes

$$
\begin{aligned}
\dot{z}_{2, i-1}= & \bar{z}_{2, i}+\tilde{f}_{2, i-1}^{[2]}\left(z_{1}, z_{2}, v\right)+c z_{1, j} z_{2, k} z_{2, l-1}+\cdots \\
\dot{\bar{z}}_{2, i}= & z_{2, i+1}+\tilde{f}_{2, i}^{[2]}\left(z_{1}, z_{2}, v\right) \\
& -c\left(\lambda_{j} z_{1, j}+z_{1, j+}\right) z_{2, k} z_{2, l-1} \\
& -c z_{2, j} z_{2, k+1} z_{2, l-1}+\cdots
\end{aligned}
$$

and all the other cubic terms remain the same. Recall that the symbol $z_{1, j+}$ denotes $z_{1, j+1}$ if

$$
\dot{z}_{1, j}=\lambda_{j} z_{1, j}+z_{1, j+1}
$$

and denotes 0 if

$$
\dot{z}_{1, j}=\lambda_{j} z_{1, j}
$$

If $k=l-1$, we can pull up the cubic term by defining

$$
\bar{z}_{2, i}=z_{2, i}-\frac{c}{2} z_{1, j} z_{2, k} z_{2, k}
$$

then in the new terms generated by the transformation, the two largest indices of $z_{2, k}$ are identical.

By pulling up all the cubic terms until $l=k$, we obtain

$$
\begin{aligned}
\dot{z}_{2, i}=z_{2, i+1}+\tilde{f}_{2, i}^{[2]}\left(z_{1}, z_{2}, v\right) & \\
& +\sum_{j=1}^{n_{1}} \sum_{k=1}^{n_{2}+1} \zeta_{i}^{j k k} z_{1, j} z_{2, k}^{2}+\cdots
\end{aligned}
$$

which is almost the normal form (3.8).

By pushing down, we can make $\zeta_{i}^{j k k}=0$ for $1 \leq k \leq i+1$. Consider a piece of the dynamics

$$
\begin{aligned}
\dot{z}_{2, i} & =z_{2, i+1}+\tilde{f}_{2, i}^{[2]}\left(z_{1}, z_{2}, v\right)+c z_{1, j} z_{2, k} z_{2, l}+\cdots \\
\dot{z}_{2, i+1} & =z_{2, i+2}+\tilde{f}_{2, i+1}^{[2]}\left(z_{1}, z_{2}, v\right)+\cdots
\end{aligned}
$$

If $1 \leq k \leq l \leq n_{2}$, define

$$
\bar{z}_{2, i+1}=z_{2, i+1}+c z_{1, j} z_{2, k} z_{2, l}
$$

yielding

$$
\begin{aligned}
\dot{z}_{2, i}= & \bar{z}_{2, i+1}+\tilde{f}_{2, i}^{[2]}\left(z_{1}, z_{2}, v\right)+\cdots \\
\dot{\bar{z}}_{2, i+1}= & z_{2, i+2}+\tilde{f}_{2, i+1}^{[2]}\left(z_{1}, z_{2}, v\right)+c\left(\lambda_{j} z_{2, j}+z_{2, j+}\right) z_{2, k} z_{2, l} \\
& +c z_{1, j} z_{2, k+1} z_{2, l}+c z_{1, j} z_{2, k} z_{2, l+1}+\cdots
\end{aligned}
$$

and all the other cubic terms remain unchanged. Notice that if $i+1=n_{2}$ then we can absorb the cubic terms into the control using feedback. The terms in (3.17) where $1 \leq j \leq k \leq l \leq$ 
$i+1$ can be repeatedly pushed down and absorbed in the control. The result is (3.8).

We defer to later the proof that $\zeta_{i}^{j k k}$ is given by (3.14) and is an invariant

$$
\tilde{f}_{2}^{[2 ; 1]}\left(z_{1} ; z_{2}, v\right)
$$

Consider a part of the dynamics

$$
\begin{aligned}
\dot{z}_{2, i-1} & =z_{2, i}+\tilde{f}_{2, i-1}^{[2]}\left(z_{1}, z_{2}, v\right)+\cdots \\
\dot{z}_{2, i} & =z_{2, i+1}+\tilde{f}_{2, i}^{[2]}\left(z_{1}, z_{2}, v\right)+c z_{1, j} z_{1, k} z_{2, l}+\cdots
\end{aligned}
$$

where $1<i \leq n_{2}, 1 \leq j \leq k \leq n_{1}, 1 \leq l \leq n_{2}+1$.

If $1<l$, we can pull up the cubic term by defining

$$
\bar{z}_{2, i}=z_{2, i}-c z_{1, j} z_{1, k} z_{2, l-1}
$$

then in the new terms generated by the transformation, the largest index of $z_{2, s}$ is $s=l-1$. So by pulling up all cubic terms until $l=1$, we obtain

$$
\dot{z}_{2, i}=z_{2, i+1}+\tilde{f}_{2, i}^{[2]}\left(z_{1}, z_{2}, v\right)+\bar{c} z_{1, j} z_{1, k} z_{2,1}+\cdots
$$

Repeated pushing down eliminates this term. Consider a part of the dynamics

$$
\begin{aligned}
\dot{z}_{2, i} & =z_{2, i+1}+\tilde{f}_{2, i}^{[2]}\left(z_{1}, z_{2}, v\right)+c z_{1, j} z_{1, k} z_{2, l}+\cdots \\
\dot{z}_{2, i+1} & =z_{2, i+2}+\tilde{f}_{2, i+1}^{[2]}\left(z_{1}, z_{2}, v\right)+\cdots
\end{aligned}
$$

where $1 \leq l \leq i \leq n_{2}$. Define

$$
\bar{z}_{2, i+1}=z_{2, i+1}+c z_{1, j} z_{1, k} z_{2, l}
$$

yielding

$$
\begin{aligned}
\dot{z}_{2, i}= & \bar{z}_{2, i+1}+\tilde{f}_{2, i}^{[2]}\left(z_{1}, z_{2}, v\right)+\cdots \\
\dot{\bar{z}}_{2, i+1}= & z_{2, i+2}+\tilde{f}_{2, i+1}^{[2]}\left(z_{1}, z_{2}, v\right) \\
& +c\left(\lambda_{j} z_{1, j}+z_{1, j+}\right) z_{1, k} z_{2, l} \\
& +z_{1, j}\left(\lambda_{k} z_{1, k}+z_{1, k+}\right) z_{2, l}+c z_{1, j} z_{1, k} z_{2, l+1}+\cdots
\end{aligned}
$$

and all the other cubic terms remain unchanged. We can continue to push down until $i+1=n_{2}$ and the cubic term can be absorbed into the control using feedback. The result is $\tilde{f}_{2}^{[2 ; 1]}\left(z_{1} ; z_{2}, v\right)=0$

$$
\tilde{f}_{2}^{[3 ; 0]}\left(z_{1} ; z_{2}, v\right)
$$

This part is similar to the proof of $\tilde{f}_{2}^{[2 ; 0]}$. The cubic term can be pushed down repeatedly by

$$
\bar{z}_{2, i+1}=z_{2, i+1}+c z_{1, j} z_{1, k} z_{1, l}
$$

until $i+1=n$ and it can be absorbed in the control via feedback. The result is $\tilde{f}_{2}^{[3 ; 0]}\left(z_{1} ; z_{2}, v\right)=0$

$$
\tilde{f}_{1}^{[3 ; 0]}\left(z_{1} ; z_{2}, v\right) \text {. }
$$

This is just the cubic normal form of Poincare and when $A_{1}$ is diagonal $\beta_{i}^{j k l}$ are invariants; see [4], [10], [15], or [20]

$$
\tilde{f}_{1}^{[2 ; 1]}\left(z_{1} ; z_{2}, v\right) \text {. }
$$

Consider a part of the dynamics

$$
\dot{z}_{1, i}=\lambda_{i} z_{1, i}+z_{1, i+}+\tilde{f}_{1, i}^{[2]}\left(z_{1}, z_{2}, v\right)+c z_{1, j} z_{1, k} z_{2, l}+\cdots
$$

where $1 \leq i \leq n_{1}, 1 \leq j \leq k \leq n_{1}, 1 \leq l \leq n_{2}+1$.

If $l>1$, then we can pull up by defining

$$
\bar{z}_{1, i}=z_{1, i}-c z_{1, j} z_{1, k} z_{2, l-1}
$$

so that

$$
\begin{aligned}
\dot{\bar{z}}_{1, i}= & \lambda_{i} \bar{z}_{1, i}+z_{1, i+}+\tilde{f}_{1, i}^{[2]}\left(z_{1}, z_{2}, v\right)+c \lambda_{i} z_{1, j} z_{1, k} z_{2, l-1} \\
& +c\left(\lambda_{j} z_{1, j}+z_{1, j+}\right) z_{1, k} z_{2, l-1} \\
& +c z_{1, j}\left(\lambda_{k} z_{1, k}+z_{1, k+}\right) z_{2, l-1}, \ldots
\end{aligned}
$$

We can continue to pull up until $l=1$. The result is (3.5).

Next, we show that the numbers $\gamma_{i}^{j k 1}$ (3.11) are invariants when $A_{1}$ is diagonal. Clearly, they are potentially changed only by $\phi_{1}^{[2 ; 1]}\left(x_{1} ; x_{2}\right)$. Therefore, we need only consider coordinates changes of the form

$$
\bar{x}_{1, i}=x_{1, i}+c x_{1, j} x_{1, k} x_{2, l}
$$

where $1 \leq i \leq n_{1}, 1 \leq j \leq k \leq n_{1}, 1 \leq k \leq l \leq n_{2}$ because more general ones are just compositions of these. This coordinate change only affects a piece of the dynamics (2.6)

$$
\begin{aligned}
\dot{x}_{1, i}=\lambda_{i} x_{1, i}+f_{1, i}^{[2]}\left(x_{1}, x_{2}, u\right) & \\
& +f_{1, i}^{[3]}\left(x_{1}, x_{2}, u\right)+O\left(x_{1}, x_{2}, u\right)^{3}
\end{aligned}
$$

is transformed to

$$
\begin{aligned}
\dot{\bar{x}}_{1, i}= & \lambda_{i} \bar{x}_{1, i}+f_{1, i}^{[2]}\left(x_{1}, x_{2}, u\right)+f_{1, i}^{[3]}\left(x_{1}, x_{2}, u\right) \\
& +c\left(\lambda_{j}+\lambda_{k}-\lambda_{i}\right) x_{1, j} x_{1, k} x_{2, l} \\
& +c x_{1, j} x_{1, k} x_{2, l+1}+O\left(x_{1}, x_{2}, u\right)^{3} .
\end{aligned}
$$

A simple calculation shows that (3.11) is unchanged

$$
\tilde{f}_{1}^{[1 ; 2]}\left(z_{1} ; z_{2}, v\right) \text {. }
$$

Consider a part of the dynamics

$$
\dot{z}_{1, i}=\lambda_{i} z_{1, i}+z_{1, i+}+\tilde{f}_{1, i}^{[2]}\left(z_{1}, z_{2}, v\right)+c z_{1, j} z_{2, k} z_{2, l}+\cdots
$$

where $1 \leq i \leq n_{1}, 1 \leq j \leq n_{1}, 1 \leq k \leq l \leq n_{2}+1$.

If $l>1$, then we can pull up by defining

$$
\bar{z}_{1, i}=z_{1, i}-c z_{1, j} z_{2, k} z_{2, l-1}
$$

so that

$$
\begin{aligned}
\dot{\bar{z}}_{1, i}= & \lambda_{i} \bar{z}_{1, i}+z_{1, i+}+\tilde{f}_{1, i}^{[2]}\left(z_{1}, z_{2}, v\right)+c \lambda_{i} z_{1, j} z_{2, k} z_{2, l-1} \\
& -c\left(\lambda_{j} z_{1, j}+z_{1, j+}\right) z_{2, k} z_{2, l-1} \\
& -c z_{1, j} z_{2, k+1} z_{2, l-1}+\cdots
\end{aligned}
$$

We can continue to pull up until $l=k$. The result is (3.6).

We defer to later the proof that $\delta_{i}^{j k k}$ is given by (3.12) and is an invariant

$$
\tilde{f}_{1}^{[0 ; 3]}\left(z_{1} ; z_{2}, v\right) .
$$


Consider a part of the dynamics

$$
\dot{z}_{1, i}=\lambda_{i} z_{1, i}+z_{1, i+}+f_{1, i}^{[2]}\left(z_{1}, z_{2}, v\right)+c z_{2, j} z_{2, k} z_{2, l}+\cdots
$$

where $1 \leq i \leq n_{1}, 1 \leq j \leq k \leq l \leq n_{2}+1$.

If $k<l-1$, then we can pull up by defining

$$
\bar{z}_{1, i}=z_{1, i}-c z_{2, j} z_{2, k} z_{2, l-1} .
$$

If $k=l-1$, then we can pull up by defining

$$
\bar{z}_{1, i}=z_{1, i}-\frac{c}{2} z_{2, j} z_{2, k} z_{2, k}
$$

We can continue to pull up until $l=k$. The result is (3.7).

Assume that the system in quadratic normal form (2.6) with coordinates $x_{1}, x_{2}, u$. We show that the numbers $\delta_{i}^{j k k}, \epsilon_{i}^{j k k}, \zeta_{i}^{j k k}, \eta_{i}^{j k k}$ as given by (3.12)-(3.15) are invariants under cubic change of coordinates (3.18) and cubic feedback (3.19)

$$
\begin{aligned}
{\left[\begin{array}{l}
z_{1} \\
z_{2}
\end{array}\right] } & =\left[\begin{array}{l}
x_{1} \\
x_{2}
\end{array}\right]-\left[\begin{array}{l}
\phi_{1}^{[3]}\left(x_{1}, x_{2}\right) \\
\phi_{2}^{[3]}\left(x_{1}, x_{2}\right)
\end{array}\right] \\
v & =u-\alpha_{1}^{[3]}\left(x_{1}, x_{2}, u\right) .
\end{aligned}
$$

Under the cubic feedback (3.19), the control is transformed from $u$ to $v$. Let $G=(\partial / \partial u)$ and $\bar{G}=(\partial / \partial v)$. By the chain rule

$$
G=\frac{\partial v}{\partial u} \bar{G}=\left(1+O\left(x_{1}, x_{2}, u\right)^{2}\right) \bar{G} .
$$

It is straightforward to show by induction that for $0 \leq k \leq n_{2}$

$$
\begin{aligned}
(-1)^{k} \mathbf{a d}_{F}^{k}(G) & =\frac{\partial}{\partial x_{2, n_{2}+1-k}}+O\left(x_{1}, x_{2}, u\right) \frac{\partial}{\partial x} \\
(-1)^{n_{2}+1} \mathbf{a d}_{F}^{n_{2}+1}(G) & =O\left(x_{1}, x_{2}, u\right) \frac{\partial}{\partial x}
\end{aligned}
$$

and

$$
\begin{aligned}
\operatorname{ad}_{F}^{k}(G)= & \operatorname{ad}_{F}^{k}(\bar{G})+\sum_{r=0}^{k} O\left(x_{1}, x_{2}, u\right)^{2} \\
& \times \frac{\partial}{\partial x_{2, n_{2}+1-r}}+O\left(x_{1}, x_{2}, u\right)^{3} \frac{\partial}{\partial x} \\
\operatorname{ad}_{F}^{n_{2}+1}(G)= & \operatorname{ad}_{F}^{n_{2}+1}(\bar{G})+\sum_{r=0}^{n_{2}} O\left(x_{1}, x_{2}, u\right)^{2} \\
& \times \frac{\partial}{\partial x_{2, n_{2}+1-r}}+O\left(x_{1}, x_{2}, u\right)^{3} \frac{\partial}{\partial x}
\end{aligned}
$$

so

$$
\begin{aligned}
& {\left[\operatorname{ad}_{F}^{k+1}(G), \mathbf{a d}_{F}^{k}(G)\right]=\left[\operatorname{ad}_{F}^{k+1}(\bar{G}), \mathbf{a d}_{F}^{k}(\bar{G})\right]} \\
& +\sum_{r=0}^{k+1} O\left(x_{1}, x_{2}, u\right) \frac{\partial}{\partial x_{2, n_{2}+1-r}}+O\left(x_{1}, x_{2}, u\right)^{2} \frac{\partial}{\partial x} .
\end{aligned}
$$

Therefore, for $0 \leq k \leq n_{2}$ and $1 \leq i \leq n_{1}$

$$
\begin{aligned}
{\left[\operatorname{ad}_{F}^{k+1}(G),\right.} & \left.\operatorname{ad}_{F}^{k}(G)\right]\left(x_{1, i}\right) \\
& =\left[\operatorname{ad}_{F}^{k+1}(\bar{G}), \mathbf{a d}_{F}^{k}(\bar{G})\right]\left(x_{1, i}\right)+O\left(x_{1}, x_{2}, u\right)^{2} .
\end{aligned}
$$

For $0 \leq k \leq n_{2}$ and $1 \leq i \leq n_{2}-1-k$

$$
\begin{aligned}
& {\left[\operatorname{ad}_{F}^{k+1}(G), \mathbf{a d}_{F}^{k}(G)\right]\left(x_{2, i}\right)} \\
& \quad=\left[\operatorname{ad}_{F}^{k+1}(\bar{G}), \mathbf{a d}_{F}^{k}(\bar{G})\right]\left(x_{2, i}\right)+O\left(x_{1}, x_{2}, u\right)^{2} .
\end{aligned}
$$

Hence, for $1 \leq j \leq k \leq n_{2}+1$ and $1 \leq i \leq n_{1}$

$$
\begin{aligned}
& {\left[\operatorname{ad}_{F}^{n_{2}+1-j}(G),\left[\operatorname{ad}_{F}^{n_{2}+2-k}(G),\right.\right.} \\
& \left.\left.\operatorname{ad}_{F}^{n_{2}+1-k}(G)\right]\right]\left(x_{1, i}\right)(0,0,0) \\
& =\left[\operatorname{ad}_{F}^{n_{2}+1-j}(\bar{G}),\left[\operatorname{ad}_{F}^{n_{2}+2-k}(\bar{G}),\right.\right. \\
& \left.\left.\quad \operatorname{ad}_{F}^{n_{2}+1-k}(\bar{G})\right]\right]\left(x_{1, i}\right)(0,0,0) .
\end{aligned}
$$

For $1 \leq j \leq k \leq n_{2}+1$ and $1 \leq i \leq k-2$

$$
\begin{aligned}
& {\left[\operatorname{ad}_{F}^{n_{2}+1-j}(G),\left[\operatorname{ad}_{F}^{n_{2}+2-k}(G),\right.\right.} \\
& \left.\left.\operatorname{ad}_{F}^{n_{2}+1-k}(G)\right]\right]\left(x_{2, i}\right)(0,0,0) \\
& =\left[\operatorname{ad}_{F}^{n_{2}+1-j}(\bar{G}),\left[\operatorname{ad}_{F}^{n_{2}+2-k}(\bar{G}),\right.\right. \\
& \left.\left.\quad \operatorname{ad}_{F}^{n_{2}+1-k}(\bar{G})\right]\right]\left(x_{2, i}\right)(0,0,0) .
\end{aligned}
$$

Since $z=x+O(x)^{3}$, by the chain rule

$$
\frac{\partial}{\partial x_{1, j}}=\frac{\partial}{\partial z_{1, j}}+O\left(x_{1}, x_{2}, u\right)^{2} \frac{\partial}{\partial x} .
$$

Hence, for $1 \leq j \leq n_{1}, 1 \leq k \leq n_{2}+1$ and $1 \leq i \leq n_{1}$

$$
\begin{aligned}
& {\left[\frac{\partial}{\partial x_{1, j}},\left[\operatorname{ad}_{F}^{n_{2}+2-k}(G), \mathbf{a d}_{F}^{n_{2}+1-k}(G)\right]\right]\left(x_{1, i}\right)(0,0,0)} \\
& \quad=\left[\frac{\partial}{\partial z_{1, j}},\left[\operatorname{ad}_{F}^{n_{2}+2-k}(\bar{G}), \mathbf{a d}_{F}^{n_{2}+1-k}(\bar{G})\right]\right]\left(x_{1, i}\right)(0,0,0) .
\end{aligned}
$$

For $1 \leq j \leq n_{1}, 1 \leq k \leq n_{2}+1$ and $1 \leq i \leq k-2$

$$
\begin{aligned}
& {\left[\frac{\partial}{\partial x_{1, j}},\left[\operatorname{ad}_{F}^{n_{2}+2-k}(G), \mathbf{a d}_{F}^{n_{2}+1-k}(G)\right]\right]\left(x_{2, i}\right)(0,0,0)} \\
& \quad=\left[\frac{\partial}{\partial z_{1, j}},\left[\operatorname{ad}_{F}^{n_{2}+2-k}(\bar{G}), \mathbf{a d}_{F}^{n_{2}+1-k}(\bar{G})\right]\right]\left(x_{2, i}\right)(0,0,0) .
\end{aligned}
$$

We have shown that (3.12)-(3.15) are invariant under cubic feedback (3.19). Since $z_{i}=x_{i}+O(x)^{3}$ they are also invariant under cubic change of coordinates (3.18).

It remains to show that if a system is in cubic normal form (3.3) then $\delta_{i}^{j k k}, \epsilon_{i}^{j k k}, \zeta_{i}^{j k k}, \eta_{i}^{j k k}$ are given by (3.12)-(3.15). Define the partial differential operators

$$
\begin{aligned}
& \bar{F}=\sum_{i=1}^{2} \sum_{j=1}^{n_{i}} \tilde{f}_{i, j}\left(z_{1}, z_{2}, v\right) \frac{\partial}{\partial z_{i, j}} \\
& \bar{G}=\frac{\partial}{\partial v}
\end{aligned}
$$

where $\tilde{f}$ is the right side of (3.3). 
By induction, one can show that for $1 \leq k \leq n_{2}, l=n_{2}+$ $1-k$

$$
\begin{aligned}
& (-1)^{k} \operatorname{ad}_{\bar{F}}^{k}(\bar{G})=\frac{\partial}{\partial z_{2, l}}+2 \sum_{i=1}^{n_{1}} \delta_{i}^{l+1, l+1} z_{2, l+1} \frac{\partial}{\partial z_{1, i}} \\
& +2 \sum_{i=1}^{l-1} \epsilon_{i}^{l+1, l+1} z_{2, l+1} \frac{\partial}{\partial z_{2, i}} \\
& +O\left(z_{1}, z_{2, l+2}, \ldots, z_{2, n_{2}+1}\right) \frac{\partial}{\partial z} \\
& +2 \sum_{i=1}^{n_{1}} \sum_{j=1}^{n_{1}} \delta_{i}^{j, l+1, l+1} z_{1, j} z_{2, l+1} \frac{\partial}{\partial z_{1, i}} \\
& +2 \sum_{i=1}^{n_{1}} \sum_{j=1}^{l} \epsilon_{i}^{j, l+1, l+1} z_{2, j} z_{2, l+1} \frac{\partial}{\partial z_{1, i}} \\
& +3 \sum_{i=1}^{n_{1}} \epsilon_{i}^{l+1, l+1, l+1} z_{2, l+1} z_{2, l+1} \frac{\partial}{\partial z_{1, i}} \\
& +2 \sum_{i=1}^{l-1} \sum_{j=1}^{n_{1}} \zeta_{i}^{j, l+1, l+1} z_{1, j} z_{2, l+1} \frac{\partial}{\partial z_{2, i}} \\
& +2 \sum_{i=1}^{l-1} \sum_{j=1}^{l} \eta_{i}^{j, l+1, l+1} z_{2, j} z_{2, l+1} \frac{\partial}{\partial z_{2, i}} \\
& +3 \sum_{i=1}^{l-1} \eta_{i}^{l+1, l+1, l+1} z_{2, l+1} z_{2, l+1} \frac{\partial}{\partial z_{2, i}} \\
& +O(z)^{3} \frac{\partial}{\partial z} \\
& (-1)^{n_{2}+1} \mathbf{a d}_{\bar{F}}^{n_{2}+1}(\bar{G})=2 \sum_{i=1}^{n_{1}} \delta_{i}^{11} z_{2,1} \frac{\partial}{\partial z_{1, i}} \\
& +O\left(z_{1}, z_{2,2}, \ldots, z_{2, n_{2}+1}\right) \frac{\partial}{\partial z} \\
& +2 \sum_{i=1}^{n_{1}} \sum_{j=1}^{n_{1}} \delta_{i}^{j, 1,1} z_{1, j} z_{2,1} \frac{\partial}{\partial z_{1, i}} \\
& +3 \sum_{i=1}^{n_{1}} \epsilon_{i}^{1,1,1} z_{2,1} z_{2,1} \frac{\partial}{\partial z_{1, i}} \\
& +O(z)^{3} \frac{\partial}{\partial z} \text {. }
\end{aligned}
$$

Then, for $l=n_{2}+1-k$

$$
\begin{aligned}
{\left[\operatorname{ad}_{\bar{F}}^{k+1}(\bar{G}), \operatorname{ad}_{\bar{F}}^{k}(\bar{G})\right]=} & +2 \sum_{i=1}^{n_{1}} \delta_{i}^{l, l} \frac{\partial}{\partial z_{1, i}} \\
& +2 \sum_{i=1}^{l-2} \epsilon_{i}^{l, l} \frac{\partial}{\partial z_{2, i}} \\
& +2 \sum_{i=1}^{n_{1}} \sum_{j=1}^{n_{1}} \delta_{i}^{j, l, l} z_{1, j} \frac{\partial}{\partial z_{1, i}} \\
& +2 \sum_{i=1}^{n_{1}} \sum_{j=1}^{l-1} \epsilon_{i}^{j, l, l} z_{2, j} \frac{\partial}{\partial z_{1, i}} \\
& +6 \sum_{i=1}^{n_{1}} \epsilon_{i}^{l, l, l} z_{2, l} \frac{\partial}{\partial z_{1, i}}
\end{aligned}
$$

$$
\begin{aligned}
& +2 \sum_{i=1}^{l-2} \sum_{j=1}^{n_{1}} \zeta_{i}^{j, l, l} z_{1, j} \frac{\partial}{\partial z_{2, i}} \\
& +2 \sum_{i=1}^{l-2} \sum_{j=1}^{l-1} \eta_{i}^{j, l, l} z_{2, j} \frac{\partial}{\partial z_{2, i}} \\
& +6 \sum_{i=1}^{l-2} \eta_{i}^{l, l, l} z_{2, l} \frac{\partial}{\partial z_{2, i}} \\
& +O\left(z_{2, l+1}, \ldots, z_{2, n_{2}+1}\right) \frac{\partial}{\partial z} \\
& +O(z)^{2} \frac{\partial}{\partial z}
\end{aligned}
$$

which confirms (3.12)-(3.15).

Remarks: Again, it is not essential that $A_{2}, B_{2}$ be in Brunovsky form, all they need to be is in controller companion form, i.e.,

$$
\begin{aligned}
A_{2} & =\left[\begin{array}{ccccc}
0 & 1 & 0 & \cdots & 0 \\
0 & 0 & 1 & \cdots & 0 \\
& & \ddots & \ddots & \\
0 & 0 & 0 & \cdots & 1 \\
a_{n, 1} & a_{n, 2} & a_{n, 3} & \cdots & a_{n, n}
\end{array}\right] \\
B_{2} & =\left[\begin{array}{c}
0 \\
0 \\
\vdots \\
0 \\
b_{n}
\end{array}\right] .
\end{aligned}
$$

The cubic normal form (3.3)-(3.9) remains the same but the moduli (3.10)-(3.15) are different.

In the aforementioned normal form, the cubic terms which are not removable have been pulled up as far as possible, i.e., to move the two largest indices in $x_{2, j} x_{2, k} x_{2, l}$ together. One can also push down the unremovable terms down to obtain the following normal form:

$$
\begin{aligned}
& {\left[\begin{array}{c}
\dot{z}_{1} \\
\dot{z}_{2}
\end{array}\right]=\left[\begin{array}{cc}
A_{1} & 0 \\
0 & A_{2}
\end{array}\right]\left[\begin{array}{c}
z_{1} \\
z_{2}
\end{array}\right]+\left[\begin{array}{c}
0 \\
B_{2}
\end{array}\right] v} \\
& +\left[\begin{array}{ccccc}
\hat{f}_{1}^{[2 ; 0]} & + & \bar{f}_{1}^{[1 ; 1]} & + & \bar{f}_{1}^{[0 ; 2]} \\
0 & + & 0 & + & \bar{f}_{2}^{0 ; 2]}
\end{array}\right]\left(z_{1}, z_{2}, v\right)
\end{aligned}
$$

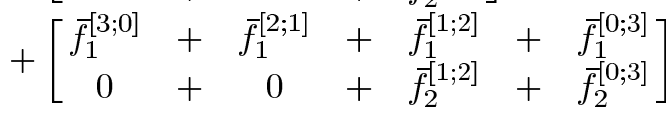

$$
\begin{aligned}
& \times\left(z_{1}, z_{2}, v\right) \\
& +O\left(z_{1}, z_{2}, v\right)^{4}
\end{aligned}
$$

where the quadratic terms are given by (2.21) and

$$
\begin{aligned}
\bar{f}_{1}^{[3 ; 0]} & =\tilde{f}_{1}^{[3 ; 0]} \\
\vec{f}_{1}^{[2 ; 1]} & =\tilde{f}_{1}^{[2 ; 1]} \\
\vec{f}_{1}^{[1 ; 2]} & =\tilde{f}_{1}^{[1 ; 2]} \\
\vec{f}_{1}^{[0 ; 3]} & =\tilde{f}_{1}^{[0 ; 3]} \\
\vec{f}_{2}^{[1 ; 2]} & =\sum_{i=1}^{n_{2}-1} \sum_{j=1}^{n_{1}} \sum_{k=n_{2}-i+2}^{n_{2}+1} \zeta_{i}^{j, k, n_{2}+1} \mathbf{e}^{2, i} z_{1, j} z_{2, k} v \\
\bar{f}_{2}^{[0 ; 3]} & =\sum_{i=1}^{n_{2}-1} \sum_{k=n_{2}-i+2}^{n_{2}+1} \sum_{j=1}^{k} \eta_{i}^{j, k, n_{2}+1} \mathbf{e}^{2, i} z_{2, j} z_{2, k} v .
\end{aligned}
$$


It may not be possible to push down the cubic terms in $\tilde{f}_{1}$ because of resonances.

\section{CONTROL BifurCATIONS}

Control systems with or without parameters have continuous families of equilibria. These families can be parametrized by the set value of the control, by the value of one or more states or by any parameters in the dynamics. A control bifurcation takes place at an equilibrium where there is a loss of local, parameteric stabilizability, see the Introduction. The theory of normal forms enables us to analyze how the simplest control bifurcations can occur.

The constants in the above normal forms $\lambda_{i}, \beta_{i}^{j k}, \ldots, \eta_{i}^{j k k}$ are called moduli. Fortunately, many moduli do not affect the stabilizability of the equilibrium. Recall that in the bifurcation theory of a parametrized system of ODEs, the interesting part of the dynamics is that restricted to the center manifold. This leads to a great reduction in the dimension of the ODE that must be studied. A similar fact holds true when studying control bifurcations. In most applications one will ultimately use state feedback in an attempt to stabilize the system so the coordinates that are linearly stabilizable and their associated moduli can be ignored to a large extent. If there are modes which are neutrally stable and are not linearly stabilizable, then the particular choice of feedback will influence the shape of center manifold of the closed-loop system and the dynamics thereon. It might be possible to achieve asymptotically stable center manifold dynamics by the proper choice of feedback although it will not be exponentially stable. One can also reduce the number of moduli by considering weaker forms of equivalence than smooth change of coordinates and invertible smooth feedback. We now discuss some important bifurcations of control systems. Some of this has already been established by Kang [12].

\section{A. Fold Control Bifurcation}

Just as with classical bifurcations, the simplest control bifurcation is the fold. This control bifurcation has not been studied before. It is the only control bifurcation that is generic when the control is one dimensional. The uncontrollable part of the state is one dimensional and unstable, $n_{1}=1, A_{1}>0$. Because the linearly controllable part of the quadratic normal form (2.6) is in Brunovsky form, the equilibria $z_{e}, v_{e}$ are most conveniently parametrized by $\mu=z_{e 2,1}$. The equilibria $z_{e}(\mu), v_{e}(\mu)$ are given by

$$
\begin{aligned}
& z_{e 1,1}=-\mu^{2} A_{1}^{-1} \delta_{1}^{11}+O(\mu)^{3} \\
& z_{e 2,1}=\mu \\
& z_{e 2, i}=O(\mu)^{2} \quad i=2, \ldots, n_{2}+1 .
\end{aligned}
$$

Note that the original control system has no parameter in it. The parameter $\mu$ is introduced as the parametrization of the equilibrium set. The one-degree of freedom of the equilibrium set is introduced by the control input in the system. The local linearization around $z_{e}, v_{e}$ is

$$
\begin{aligned}
{\left[\begin{array}{l}
\dot{\tilde{z}}_{1} \\
\dot{\tilde{z}}_{2}
\end{array}\right]=\left(\left[\begin{array}{cc}
A_{1}+\mu \gamma_{1}^{11} & \mu \Delta \\
0 & A_{2}
\end{array}\right]+O(\mu)^{2}\right) } & {\left[\begin{array}{c}
\tilde{z}_{1} \\
\tilde{z}_{2}
\end{array}\right] } \\
& +\left(\left[\begin{array}{c}
0 \\
B_{2}
\end{array}\right]+O(\mu)^{2}\right) \tilde{v}
\end{aligned}
$$

where $\tilde{z}=z-z_{e}(\mu), \tilde{v}=v-v_{e}(\mu)$, and $\Delta=$ $\left[\begin{array}{llll}2 \delta_{1}^{11} & 0 & \ldots & 0\end{array}\right]$.

If the transversality condition $\delta_{1}^{11} \neq 0$ is satisfied, then about any equilibrium except $\mu=0$; the system is linearly controllable hence stabilizable. However, it is not locally, parameterically, smoothly stabilizable at $\mu=0$. Consider a parametrized family of feedbacks $v=\kappa(z, \mu)$ of the form

$$
\tilde{v}=K_{1}(\mu) \tilde{z_{1}}+K_{2}(\mu) \tilde{z_{2}} \text {. }
$$

One would like to find a continuous family of feedbacks that makes the family of equilibria asymptotically stable, i.e., for each small $\mu$, the closed-loop system $\dot{z}=\tilde{f}(z, \kappa(z, \mu))$ is asymptotically stable to $z_{e}(\mu)$. The lowest degree terms of more general smooth feedbacks will be like (4.1).

Clearly, the $z_{2}$ subsystem is stabilizable for all $\mu$ by proper choice of $K_{2}$ and this gain can be chosen independent of $\mu$. The question is can we find $K_{1}(\mu)$ which stabilizes the $z_{1}$ coordinate for all small $|\mu|$.

Since the linear approximations are stabilizable for $\mu \neq 0$, it is certainly possible to find a stabilizing feedback at each such $\mu$. The linear approximation at $\mu=0$ has an uncontrollable, unstable mode so it is not possible to stabilize it. However, is it possible to stabilize the approximations for $\mu \neq 0$ with a feedback that is continuous through $\mu=0$ ? The answer is no for systems with a fold control bifurcation. For any continuous feedback, the closed-loop system will be unstable in some neighborhood of $\mu=0$.

The closed-loop linear approximation

$$
\left[\begin{array}{l}
\dot{\tilde{z}}_{1} \\
\tilde{z}_{2}
\end{array}\right]=\left(\left[\begin{array}{cc}
A_{1}+\mu \gamma_{1}^{11} & \mu \Delta \\
B_{2} K_{1} & A_{2}+B_{2} K_{2}
\end{array}\right]+O(\mu)^{2}\right)\left[\begin{array}{c}
\tilde{z}_{1} \\
\tilde{z}_{2}
\end{array}\right]
$$

is clearly unstable at $\mu=0$ since $A_{1}>0$. Furthermore if the feedback $v=K_{2}(\mu) z_{2}$ stabilizes the $z_{2}$ subsystem then $A_{1}$ is a simple positive root of the characteristic polynomial of the closed-loop system when $\mu=0$. Hence, there is a positive root of the characteristic polynomial for small $|\mu|$.

By using higher and higher gain, it is possible to stabilize the system closer and closer to $\mu=0$. But if the feedback (4.1) is continuous in $\mu$, at best it will stabilize only over an interval of values of $\mu$ that lies wholly to the left or right of $\mu=0$. If a smooth family of feedbacks does stabilize the system for some $\mu$ close to zero, the parametrized closed loop system generically undergoes a classical fold bifurcation (also called a saddle-node bifurcation) at some $\mu$ closer to zero.

We illustrate this with a simple example in normal form

$$
\begin{aligned}
& \dot{z}_{1}=z_{1}-z_{2}^{2} \\
& \dot{z}_{2}=v .
\end{aligned}
$$


The equilibria are $z_{e, 1}=\mu^{2}, z_{e, 2}=\mu, v_{e}=0$. Under the feedback $v=K_{1}(\mu) \tilde{z}_{1}+K_{2}(\mu) \tilde{z}_{2}$, the closed-loop linear approximation is

$$
\left[\begin{array}{l}
\dot{\tilde{z}}_{1} \\
\dot{\tilde{z}}_{2}
\end{array}\right]=\left[\begin{array}{cc}
1 & -2 \mu \\
K_{1}(\mu) & K_{2}(\mu)
\end{array}\right]\left[\begin{array}{l}
\tilde{z}_{1} \\
\tilde{z}_{2}
\end{array}\right]
$$

where $\tilde{z}=z-z_{e}(\mu), \tilde{v}=v-v_{e}(\mu)$. This is asymptotically stable iff the trace is negative and the determinant is positive, which yields the inequalities $-2 \mu K_{1}(\mu)<K_{2}(\mu)<-1$. Clearly, there is no bounded $K_{1}(\mu)$ that satisfies these for all small $|\mu|$.

If we choose $K_{1}=10$ and $K_{2}=-2$ then the closed-loop linear approximation is stable for $\mu>0.1$ and unstable for $\mu<0.1$. It undergoes a fold bifurcation at $\mu=0.1$. If we choose $K_{1}=-10$ and $K_{2}=-2$ then the closed loop linear approximation is stable for $\mu<-0.1$ and unstable for $\mu>$ -0.1 . It undergoes a fold bifurcation at $\mu=-0.1$.

To see this, consider the closed-loop nonlinear system under the former feedback, $K_{1}=10$ and $K_{2}=-2$, in coordinates centered at the bifurcation, $\bar{z}_{1}=z_{1}-0.01, \bar{z}_{2}=z_{2}-0.1, \bar{\mu}=$ $\mu-0.1$,

$$
\begin{aligned}
& \dot{\bar{z}}_{1}=\bar{z}_{1}-0.2 \bar{z}_{2}-\bar{z}_{2}^{2} \\
& \dot{\bar{z}}_{2}=10 \bar{z}_{1}-2 \bar{z}_{2}-10 \bar{\mu}^{2} .
\end{aligned}
$$

It is convenient to reparametrize by $\nu=\bar{\mu}^{2} \geq 0$. The center manifold is given by

$$
\bar{z}_{2}=10 \nu+5 \bar{z}_{1}+2500 \nu^{2}-1000 \nu \bar{z}_{1}+125 \bar{z}_{1}^{2}+O\left(\bar{z}_{1}, \nu\right)^{3}
$$

and the center manifold dynamics is

$$
\dot{\bar{z}}_{1}=2 \nu-600 \nu^{2}+300 \nu \bar{z}_{1}-500 \bar{z}_{1}^{2}+O\left(\bar{z}_{1}, \nu\right)^{3}
$$

or in the variables $\hat{z}_{1}=\sqrt{50}\left(\bar{z}_{1}-3 \nu\right), \hat{\nu}=2 \nu-150 \nu^{2}$

$$
\dot{\hat{z}}_{1}=\hat{\nu}-\hat{z}_{1}^{2}+O\left(\hat{z}_{1}, \hat{\nu}\right)^{3}
$$

the familiar form of a fold bifurcation.

\section{B. Transcritical Control Bifurcation}

Kang [12] studied this control bifurcation but not under this name. This is the degenerate case where again $n_{1}=1$, but $A_{1}=0$. Hence, this control bifurcation is not of codimension one. Kang showed that the local behavior is determined by the roots of quadratic form

$$
\beta_{1}^{11} z_{1,1}^{2}+\gamma_{1}^{11} z_{1,1} z_{2,1}+\delta_{1}^{11} z_{2,1}^{2}=0 .
$$

If this form is positive or negative definite, then there is only an isolated equilibrium which is unstabilizable. If it is indefinite but not degenerate, there are two curves of equilibria which cross. For example, suppose $\beta_{1}^{11}=1, \gamma_{1}^{11}=0, \delta_{1}^{11}=-1$ and all the other nonlinear terms are zero. Then, the equilibria are

$$
\begin{aligned}
& z_{e 1,1}^{ \pm}= \pm \mu \\
& z_{e 2,1}=\mu \\
& z_{e 2, i}=0 \quad i=2, \ldots, n_{2}+1 .
\end{aligned}
$$

Let $z_{e}(\mu), v_{e}(\mu)$ be one smooth curve of equilibria, e.g., $z_{e 1}^{+}$, the local linearizations around it are

$$
\begin{aligned}
{\left[\begin{array}{c}
\dot{\tilde{z}}_{1} \\
\tilde{\tilde{z}}_{2}
\end{array}\right]=\left(\left[\begin{array}{cc}
2 \mu & \mu \Delta \\
0 & A_{2}
\end{array}\right]+O(\mu)^{2}\right)\left[\begin{array}{l}
\tilde{z}_{1} \\
\tilde{z}_{2}
\end{array}\right] } \\
+\left(\left[\begin{array}{c}
0 \\
B_{2}
\end{array}\right]+O(\mu)^{2}\right) \tilde{v}
\end{aligned}
$$

where $\tilde{z}=z-z_{e}(\mu), \tilde{v}=v-v_{e}(\mu)$, and $\Delta=\left[\begin{array}{llll}-2 & 0 & \ldots & 0\end{array}\right]$.

Suppose we close the loop with the smooth feedback

$$
\tilde{v}=K_{1}\left(z_{1}-z_{e 1}\right)+K_{2}\left(z_{2}-z_{e 2}\right)
$$

where $K_{1}, K_{2}$ are constants chosen to stabilize the + branch of equilibria

$$
\begin{aligned}
& z_{e 1,1}=\mu \\
& z_{e 2,1}=\mu \\
& z_{e 2, i}=0, \quad i=2, \ldots, n_{2}+1
\end{aligned}
$$

for $\mu>0$. The linear approximations of the closed-loop dynamics around these equilibria are

$$
\left[\begin{array}{l}
\dot{\tilde{z}}_{1} \\
\dot{\tilde{z}}_{2}
\end{array}\right]=\left(\left[\begin{array}{cc}
2 \mu & \mu \Delta \\
B_{2} K_{1} & A_{2}+B_{2} K_{2}
\end{array}\right]+O(\mu)^{2}\right)\left[\begin{array}{l}
\tilde{z}_{1} \\
\tilde{z}_{2}
\end{array}\right]
$$

which has a zero eigenvalue at $\mu=0$. If $K_{1}+K_{21}=0$ then zero is an eigenvalue for all $\mu$ which contradicts our assumption that the feedback (4.2) stabilizes the equilibria for $\mu>0$. Hence, we assume that $K_{1}+K_{21} \neq 0$, then the determinant of the full system matrix changes sign at $\mu=0$ so there is a change of stability and the closed-loop system is unstable at the equilibria where $\mu<0$.

The closed-loop system has another branch of equilibria. If $K_{1} \neq K_{21}$, then these equilibria are given by

$$
\begin{aligned}
z_{e 1,1} & =\frac{K_{1}+K_{21}}{K_{1}-K_{21}} \mu \\
z_{e 2,1} & =-\frac{K_{1}+K_{21}}{K_{1}-K_{21}} \mu \\
z_{e 2, i} & =0 \quad i=2, \ldots, n_{2} .
\end{aligned}
$$

and the linear approximations of the closed-loop dynamics around these equilibria are

$$
\left[\begin{array}{c}
\dot{\tilde{z}}_{1} \\
\dot{\tilde{z}}_{2}
\end{array}\right]=\left(\left[\begin{array}{cc}
2 \frac{K_{1}+K_{21}}{K_{1}-K_{21}} \mu & -\frac{K_{1}+K_{21}}{K_{1}-K_{21}} \mu \Delta \\
B_{2} K_{1} & A_{2}+B_{2} K_{2}
\end{array}\right]+O(\mu)^{2}\right)\left[\begin{array}{c}
\tilde{z}_{1} \\
\tilde{z}_{2}
\end{array}\right]
$$

which has a zero eigenvalue at $\mu=0$. The determinant of the full system matrix changes sign at $\mu=0$. The linear approximating dynamics is stable for $\mu<0$ and unstable for $\mu>0$ so the two branches (4.3), (4.4) of equilibria exchange stability at $\mu=0$ and the closed-loop system undergoes a transcritical bifurcation.

If $K_{1}+K_{21}=0$, then the closed-loop system has only one branch of equilibria (4.3) and the linear approximating dynamics has a zero eigenvalue for all $\mu$.

If $K_{1}-K_{21}=0$, then the closed loop system has two branches of equilibria; (4.3) where the linear approximating dynamics has a change of stability at $\mu=0$ and 
$z_{e 1,1}=-z_{e 2,1}, z_{e 2,2}=\cdots=z_{e 2, n_{2}}=0$ where the linear approximating dynamics has a zero eigenvalue for all $\mu$.

The three-state Moore and Greitzer [18] model of an axial flow compressor provides an example of a transcritical bifurcation. Feedbacks have designed to stabilize this model (see, for instance, [17]). In [13], it is proved that the Moore-Greitzer three-state model is equivalent to the following normal form:

$$
\begin{aligned}
& \dot{z}_{1,1}=-2 \sigma z_{1,1} z_{2,1}-\sigma z_{1,1}^{2}-\sigma z_{1,1} z_{2,1}^{2} \\
& \dot{z}_{2,1}=z_{2,2} \\
& \dot{z}_{2,2}=u
\end{aligned}
$$

where $\sigma$ is a constant. The eigenvalue of the uncontrollable mode is zero and its equilibrium set consists of two branches. Based on its normal form, it is proved in [13] that the system undergoes a transcritical bifurcation under linear feedback.

\section{Hopf Control Bifurcation}

This control bifurcation has not been studied before. The uncontrollable modes are a nonzero complex conjugate pair

$$
A_{1}=\left[\begin{array}{ll}
\lambda & 0 \\
0 & \bar{\lambda}
\end{array}\right]
$$

where $\lambda=\nu+i \omega, \bar{\lambda}=\nu-i \omega, \omega \neq 0$. The equilibria $z_{e}(\mu), v_{e}(\mu)$ are given by

$$
\begin{aligned}
{\left[\begin{array}{l}
z_{e 1,1} \\
z_{e 1,2}
\end{array}\right] } & =-\mu^{2} A_{1}^{-1}\left[\begin{array}{l}
\delta_{1}^{11} \\
\delta_{2}^{11}
\end{array}\right]+O(\mu)^{3} \\
z_{e 2,1} & =\mu \\
z_{e 2, i} & =O(\mu)^{2} \quad i=2, \ldots, n_{2}+1
\end{aligned}
$$

where $\delta_{1}^{11}, \delta_{2}^{11}$ is a complex conjugate pair.

The local linearization around $z_{e}, v_{e}$ is

$$
\begin{aligned}
{\left[\begin{array}{c}
\dot{\tilde{z}}_{1} \\
\dot{\tilde{z}}_{2}
\end{array}\right]=\left(\left[\begin{array}{cc}
A_{1}+\mu \Gamma & \mu \Delta \\
0 & A_{2}
\end{array}\right]+O(\mu)^{2}\right) } & {\left[\begin{array}{c}
\tilde{z}_{1} \\
\tilde{z}_{2}
\end{array}\right] } \\
& +\left(\left[\begin{array}{c}
0 \\
B_{2}
\end{array}\right]+O(\mu)^{2}\right) \tilde{v}
\end{aligned}
$$

where $\tilde{z}=z-z_{e}(\mu), \tilde{v}=v-v_{e}(\mu)$ and

$$
\Gamma=\left[\begin{array}{cc}
\gamma_{1}^{11} & \gamma_{1}^{21} \\
\gamma_{2}^{11} & \gamma_{2}^{21}
\end{array}\right] \quad \Delta=\left[\begin{array}{cccc}
2 \delta_{1}^{11} & 0 & \ldots & 0 \\
2 \delta_{2}^{11} & 0 & \ldots & 0
\end{array}\right]
$$

where $\gamma_{1}^{11}, \gamma_{2}^{11}$, and $\gamma_{1}^{21}, \gamma_{2}^{21}$ are complex conjugate pairs.

The local linearization is uncontrollable at $\mu=0$. If the transversality condition, $\Delta \neq 0$, is satisfied then the local linearization is controllable at all small $\mu \neq 0$. (This transversality condition should not be confused with the familiar transversality condition of the classical Hopf bifurcation.) If $\nu<0$, then the system is stabilizable about any equilibrium so it does not undergo a control bifurcation. If $\nu>0$, then the system is not stabilizable when $\mu=0$. If $\nu=0$, then the system is not locally, parameterically, smoothly stabilizable. The cases $\nu \geq 0$ are called Hopf control bifurcations.

If $\nu>0$ then it requires larger and larger gain to stabilize the system closer and closer to $\mu=0$. But if the feedback ((4.1)) is continuous, it will stabilize only for some small $\mu>0$ or for some small $\mu<0$ but not both. At $\mu=0$ the poles of the closed loop system are $\lambda, \bar{\lambda}$ and the poles of $A_{2}+B_{2} K_{2}(0)$.
The latter can be made stable but the former are unstable. If the feedback is bounded then as $\mu \rightarrow 0$ the poles converge to the poles of the closed loop system at $\mu=0$. The system is controllable for $\mu \neq 0$ so the poles can be placed arbitrarily by feedback. The poles associated primarily with the $z_{2}$ subsystem can be kept stable but the two poles associated primarily with the $z_{1}$ subsystem will cross into the right half plane at some small value(s) of $\mu$. Depending on the choice of feedback, they will cross one at a time as real poles, cross together through 0 or cross together as a nonzero complex conjugate pair. If they cross separately as real poles then generically the closed loop system undergoes a classical fold bifurcation as the first pole crosses. If they cross together as a nonzero complex conjugate pair then generically the system undergoes a classical Hopf bifurcation. If they cross together through zero the situation can be quite complicated and will not be discussed here.

If $\nu=0$ and the feedback (4.1) is continuous, then generically the system undergoes a Hopf bifurcation at $\mu=0$. We illustrate this with an example

$$
\begin{aligned}
\dot{x}_{1,1} & =-x_{1,2}-x_{2}^{2} \\
\dot{x}_{1,2} & =x_{1,1}-x_{2}^{2} \\
\dot{x}_{2} & =u .
\end{aligned}
$$

The equilibria are

$$
\begin{aligned}
x_{e 1,1} & =\mu^{2} \\
x_{e 1,2} & =-\mu^{2} \\
x_{e 2} & =\mu \\
u_{e} & =0
\end{aligned}
$$

and the linear approximations are

$$
\begin{aligned}
\dot{\tilde{x}}_{1,1} & =-\tilde{x}_{1,2}-2 \mu \tilde{x}_{2} \\
\dot{\tilde{x}}_{1,2} & =\tilde{x}_{1,1}-2 \mu \tilde{x}_{2} \\
\dot{\tilde{x}}_{2} & =\tilde{u}
\end{aligned}
$$

where $\tilde{x}_{1,1}=x_{1,1}-\mu^{2}, \tilde{x}_{1,2}=x_{1,2}+\mu^{2}, \tilde{x}_{2}=x_{2}-\mu, \tilde{u}=u$. The linear approximations are controllable except at $\mu=0$.

We choose the feedback $u=10 \tilde{x}_{1,1}+5 \tilde{x}_{1,2}-3 \tilde{x}_{2}$ which place the poles at $-1 \pm i,-1$ when $\mu=0.1$. Near $\mu=0$ the poles of the closed-loop linear approximations are

$$
\begin{aligned}
& s=-3+10 \mu+O(\mu)^{2} \\
& s=-5 \mu \pm i+O(\mu)^{2} .
\end{aligned}
$$

The closed-loop dynamics is

$$
\begin{aligned}
\dot{x}_{1,1} & =-x_{1,2}-x_{2}^{2} \\
\dot{x}_{1,2} & =x_{1,1}-x_{2}^{2} \\
\dot{x}_{2} & =10 x_{1,1}+5 x_{1,2}-3 x_{2}+3 \mu-5 \mu^{2} .
\end{aligned}
$$

The first Lyapunov coefficient (see [15, (10.50)]) is $l_{1}(0)=$ -70.11 . This and (4.5) imply that the closed-loop system undergoes a supercritical Hopf bifurcation at $\mu=0$. For small $\mu>0$ the origin is locally exponentially stable. For small $\mu<0$ the origin is unstable but there is a locally asymptotically stable limit cycle nearby. At $\mu=0$, the origin is locally asymptotically stable but not locally exponentially stable. 


\section{CONCLUSION}

We have presented the normal form to degree 3 of a smooth control system around an equilibrium point under the group of smooth coordinate changes and invertible smooth feedback. From these normal forms we were able to identify the simplest control bifurcations, the fold control bifurcation, the transcritical control bifurcation and the Hopf control bifurcation. We emphasize the distinction between a control bifurcation and a classical bifurcation. Bifurcation control is different from a control bifurcation. The former refers to the modification by feedback of a classical bifurcation of the uncontrolled $(u=0)$ system. Of course, these concepts are closely related and when a system with a control bifurcation is modified by smooth feedback the result is a classical bifurcation.

\section{REFERENCES}

[1] E. H. Abed and J.-H. Fu, "Local feedback stabilization and bifurcation control, Part I. Hopf bifurcations," Syst. Control Lett., vol. 7, pp. 11-17, 1986.

[2] — - "Local feedback stabilization and bifurcation control, Part II. Stationary bifurcations," Syst. Control Lett., vol. 8, pp. 467-473, 1987.

[3] E. H. Abed and H. O. Wang, "Feedback control of bifurcation and chaos in dynamical systems," in Nonlinear Dynamics and Stochastic Mechanics, S. Namachchivaya and W. Klieman, Eds. Boca Raton, FL: CRC Press, 1995, pp. 153-173.

[4] V. I. Arnold, Geometrical Methods in the Theory of Ordinary Differential Equations. New York: Springer-Verlag, 1983.

[5] J.-P. Barbot, S. Monaco, and D. Normand-Cyrot, "Quadratic forms and feedback linearization in discrete time," Int. J. Control, vol. 67, pp. 567-586, 1997

[6] D. E. Chang, W. Kang, and A. J. Krener, "Normal forms and bifurcations of control systems," presented at the 39th IEEE Conf. Decision and Control, Sydney, NSW, Australia, 2000.

[7] G. Chen, Controlling Chaos and Bifurcations in Engineering Systems. Boca Raton, FL: CRC Press, 1999.

[8] G. Chen, J. L. Moiola, and H. O. Wang, "Bifurcation control: Theories, methods, and applications," Int. J. Bifurcation Chaos, vol. 10, no. 3, pp. $511-548,2000$.

[9] O. Fitch, "The control of bifurcation with engineering applications," Ph.D. dissertation, Naval Postgraduate School, Monterey, CA, Sept. 1997.

[10] J. Guckenheimer and P. Holmes, Nonlinear Oscillations, Dynamical Systems, and Bifurcations of Vector Fields. New York: Springer-Verlag, 1983.

[11] W. Kang and A. J. Krener, "Extended quadratic controller normal form and dynamic feedback linearization of nonlinear systems," SIAM J. Control Optim., vol. 30, pp. 1319-1337, 1992.

[12] W. Kang, "Bifurcation and normal form of nonlinear control systems-Part I and part II," SIAM J. Control Optim., vol. 36, pp. 193-212, 213-232, 1998

[13] — "Bifurcation control via state feedback for systems with a single uncontrollable mode," SIAM J. Control Optim., vol. 38, pp. 1428-1452, 2000.

[14] A. J. Krener and L. Li, "Normal forms and bifurcations of discrete time nonlinear control systems," SIAM J. Control Optim., vol. 40, pp. 1697-1723, 2002.

[15] Y. A. Kuznetsov, Elements of Applied Bifurcation Theory. New York: Springer-Verlag, 1998.

[16] L. Li and A. J. Krener, "Quadratic and cubic normal forms of discrete time nonlinear control systems," in Anais do XIII Congresso Brasileiro de Automatica, CBA 2000, pp. 19-25.

[17] D.-C. Liaw and E. H. Abed, "Stability analysis and control of rotating stall," presented at the Proc. IFAC Nonlinear Control Systems Design Symp., Bordeaux, France, June 1992.
[18] F. K. Moore and E. M. Greitzer, "A theory of post-stall transients in axial compression systems-Part I: Development of equations," ASME J. Eng. Gas Turbines Power, vol. 108, pp. 68-76, 1986.

[19] I. A. Tall and W. Respondek, "Normal forms, canonical forms and invariants of single input nonlinear systems under feedback," presented at the 39th IEEE Conf. Decision and Control, Sydney, NSW, Australia, 2000.

[20] S. Wiggins, Introduction to Applied Nonlinear Dynamical Systems and Chaos. New York: Springer-Verlag, 1990.

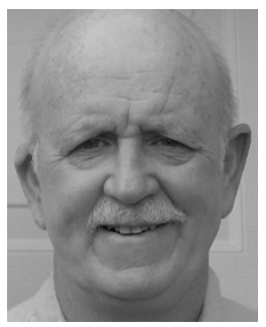

Arthur J. Krener (M'77-SM'82-F'90) was born in Brooklyn, NY, in 1942. He received the B.S. degree from Holy Cross College, Worcester, MA in 1964, and the M.A. and Ph.D. degrees from the University of California, Berkeley in 1967 and 1971, all in mathematics.

Since 1971, he has been at the University of California, Davis, where he is currently Distinguished Professor of Mathematics. He has held visiting positions at Harvard University, Cambridge, MA, the University of Rome, Rome, Italy, Imperial College of Science and Technology, London, U.K., NASA Ames Research Center, Moffett Field, CA, the University of California, Berkeley, the University of Paris IX, Paris, France, the University of Maryland, College Park, the University of Newcastle, Newcastle, Australia, and the University of Padua, Padua, Italy. His research interests are in developing methods for the control and estimation of nonlinear dynamical systems and stochastic processes.

Dr. Krener is a Fellow of the John Simon Guggenheim Foundation and a Member of the Society for Industrial and Applied Mathematics (SIAM) and the American Mathematical Society (AMS). His coauthored 1981 IEEE Transactions on Automatic Control (TAC) paper won a Best Paper Award. His coauthored 1977 TAC paper was recently chosen as one of 25 seminal papers in control.

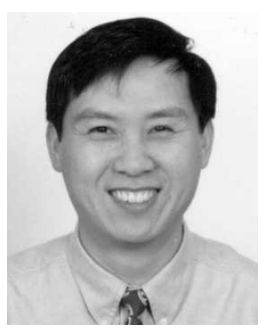

Wei Kang (S'91-M'91-SM'02) received the B.S. and M.S. degrees in mathematics from Nankai University, China in 1982 and 1985, respectively, and the Ph.D. degree in mathematics from University of California, Davis, in 1991.

$\mathrm{He}$ is an Associate Professor of Mathematics at the U.S. Naval Postgraduate School (NPS), Monterey, CA, where he has been since 1994. From 1991 to 1994, he was a Visiting Assistant Professor of Systems Science and Mathematics at Washington University, St. Louis, MO. His research interests include control systems with bifurcations, normal forms and invariants, cooperative control, industrial applications, nonlinear filtering, and nonlinear $\mathrm{H}$-infinity control. He is a Member of the Society for Industrial and Applied Mathematics (SIAM).

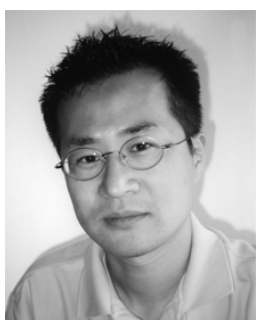

Dong Eui Chang received the Ph.D. degree in control and dynamical systems from the California Institute of Technology, Pasadena, in 2002.

He was a Postdoctoral Fellow at the University of California, Santa Barbara in 2003, and is currently a Postdoctoral Fellow at the Centre Automatic et Systemes of the Ecole des Mines de Paris, Paris, France. His research interests are in geometric mechanics and optimal control, and their application to nanotechnology and aerospace engineering.

Dr. Chang received the Society for Industrial and Applied Mathematics (SIAM) Student Paper Award in 2002. 